\title{
Endogenous discounting, wariness, and efficient capital
}

\section{taxation}

Aloisio Araujo ${ }^{* 1,2}$, Juan Pablo Gama ${ }^{\dagger 1,3}$, Rodrigo Novinski ${ }^{\ddagger 4}$, and Mario R. Pascoa $^{\S 5}$

${ }^{1}$ IMPA, Estrada dona Castorina, 110, Rio de Janeiro, 22460-320, Brazil

${ }^{2}$ FGV EPGE Brazilian School of Economics and Finance, Praia de Botafogo, 190, Rio de Janeiro, 22250-900, Brazil

${ }^{3}$ Department of Economics, Federal University of Minas Gerais, Av. Antônio Carlos, 6627, Belo Horizonte, 31270-901, Brazil

${ }^{4}$ Faculdades Ibmec, Av. Presidente Wilson, 118, Rio de Janeiro, 20030-020, Brazil

${ }^{5}$ University of Surrey, 388 Stag Hill, Guildford, GU2 7XH, United Kingdom

June 26, 2019

\begin{abstract}
When the discount factors that infinite lived consumers use at each date are not predetermined but are instead chosen within some set, depending on what the consumption plan is, impatience might not hold. More precisely, if the utility is the infimum of discounted utilities over that set of discount factor sequences, then preferences may be just upper semi-impatient. Such lack of lower semi-impatience, which we refer to as wariness, consists in neglecting distant gains but not distant losses. Examples are the precautionary case (a concern with the worst lifetime outcome) and the habit persistence case (a concern with a fall in living standards). The implementation of efficient

*aloisio@impa.br. This study was financed in part by the Coordenação de Aperfeiçoamento de Pessoal de Nível Superior - Brazil (CAPES) - Finance Code 001, the Conselho Nacional de Desenvolvimento Científico e Tecnológico - CNPq and FAPERJ in Brazil and from FCT/FEDER (project EGE-ECO/114496/2009) and NOVA FORUM in Portugal.

${ }^{\dagger}$ jpgamat@impa.br, corresponding author

${ }^{\ddagger}$ rodrigo.novinski@ibmecrj.br

§m.pascoa@surrey.ac.uk
\end{abstract}


allocations by trading assets sequentially requires taxes that avoid excessive savings by raising the opportunity of cost of saving up to the point of matching the marginal benefit of dishoarding at distant dates. Taxes on equilibrium plans are zero in many contexts.

JEL classification: D53, E40, E41, G10

Keywords: general equilibrium; monetary equilibrium; efficient taxation; endogenous discounting; transversality condition; pure charges 


\section{Introduction}

\subsection{Endogenous discounting and wariness}

This paper reexamines some core questions on the efficient consumption and assets accumulation in the light of a reformulation of the way infinite lived agents discount the future. We depart from the classical discounted utility assumption. Consumers are unsure which discount factor to use and end up choosing it within some set, depending on what the consumption plan might be. More precisely, consumers discount each consumption plan using the most adverse discount factor, the one for which the discounted utility series is the infimum of the series over that choice set. This modelling of time preferences constitutes a form, perhaps the ultimate form, of endogenous discounting. We can trace its roots to Geoffard (1996) minmax variational problem, where it was motivated on the grounds that it may be difficult to define the discount factor and, therefore, may be more intuitive to look for the utility in the worst case scenario, when the discount factor is more adverse. Non-choice theoretic forms of endogenous discounting, where the discount factor is endogenously determined from the optimal consumption plan but the resulting utility is still recursive, as in Koopmans (1960) or Uzawa (1968), were shown by Geoffard (1996) to be particular cases of his variational approach. Nice surveys of departures from traditional exogenous discounting were provided by Backus (2004) and Hansen (2004). The latter discusses also the importance of endogenous discounting for the macroeconomics literature on misspecification of preferences and robust control, as in Hansen and Sargent (2001, 2008).

The unawareness about the discount factor may be particularly interesting in the dynastic interpretation of what an infinite lived agent is: the agent may know her own discount factor but ignores how her descendants will discount the future. The worst case scenario form of endogenous discounting has important consequences for the consumers' impatience. Impatience is usually formalized in terms of the continuity of preferences with respect to 
the Mackey topology on the space of bounded consumption sequences ${ }^{1}$. Preferences are now only known to be upper semi-impatient, since the infimum of Mackey continuous utilities, over some set of discount factors, can only be shown to be upper semi-continuous. The lack of lower semi-impatience, which we refer to as wariness, means that distant losses may not be ignored, even though gains at distant dates will be.

\subsection{Wariness and pure charges in $\mathrm{AD}$ prices}

The existence Theorem 1 in Bewley (1972) ensures that Arrow-Debreu equilibrium exists, in spite of the Mackey lower-discontinuity in preferences, but the prices will be in the dual space (the space of bounded finitely additive set functions) rather than in the pre-dual (the space of summable sequences), since a pure charge may be present in the price functional. A recent work by Khan and Stinchcomb (2016) contemplates also patient preferences and $\mathrm{AD}$ prices in the dual space but questions the whole idea of discounting and uses the notion of overtaking optimality due to Weizsacker (1965) .

We show ${ }^{2}$ that $\mathrm{AD}$ prices have pure charges precisely when the the series of discounted utilities does not converge uniformly on the set of possible discount factors. Such nonuniformity occurs in two interesting cases. One case (where the infimum is taken over discount processes bounded from below by the epsilon-contamination capacity induced by a given discount factor) leads to precautionary preferences: a convex combination of a discounted utility and the infimum of the lifetime utilities. The consumer is particularly worried about the worst lifetime outcome ${ }^{3}$. The other case leads to habit persistence preferences: for another set of discount priors, we get a convex combination of a discounted utility and the infimum of average utilities up to each date. The consumer dislikes a fall

\footnotetext{
${ }^{1}$ These impatience (or myopia) notions were developed by Brown and Lewis (1981), Araujo (1985), Raut (1986) and Sawyer (1987). See also Mas-Colell and Zame (1991).

${ }^{2}$ See our Theorem 1.

${ }^{3}$ These preferences are related to the Rawlsian utility (which would be just that infimum), mentioned in Araujo (1985), but now the presence of the series of discounted utility makes preferences monotonic.
} 
in the standard of living, measured in terms of average utility up to then.

Pure charges in $\mathrm{AD}$ prices are linear functionals (on the space of bounded consumption sequences) that consist of a generalized limit (a functional assigning to each sequence a value within its liminf and its limsup, coinciding with the limit when it exists) multiplied by a scalar (the norm of the functional) which can be interpreted as the marginal utility at infinity. The latter is non-null in the two cases mentioned above when the infimum of consumption (in the precautionary case) or the infimum of consumption averages (in the habit persistence case) are attained at infinity.

\subsection{Sequential implementation}

The sequential implementation of $\mathrm{AD}$ equilibria with pure charges in prices poses a new and interesting problem.

The implementation consists in finding an allocation of initial holdings of money for which the AD consumption allocation can be attained for suitable cash balances plans that are individually optimal under the sequential budget constraints. As in any infinite horizon optimization problem, Euler and transversality conditions are necessary but might not be sufficient for individual optimality. They are sufficient once we impose portfolio constraints of the form of inequalities that are the converse to the transversality inequality ${ }^{4}$. When there are pure charges in the supergradients of the utility function evaluated at the AD net trade, such portfolio constraints are non-trivial even though money cannot be shorted. In fact, the constraints require the marginal benefit from dishoarding at infinity not to exceed the accumulated opportunity cost of hoarding along the lifetime.

Instead of imposing directly such portfolio constraints, we introduce taxes on money balances that raise the cost of holding cash and eliminate the benefits-costs gap that would be ruled out by the constraints. Such taxes can actually be defined recursively, on the basis

\footnotetext{
${ }^{4}$ For example, under impatience and if the asset could be shorted, the transversality condition says that, for the optimal plan, the limiting deflated position has to be non-positive, while the portfolio constraints should require that limit to be non-negative (and, thereby, prevent Ponzi schemes).
} 
of what are the accumulated hoarding cost and the dishoarding benefit at each moment in time. Moreover, in many interesting contexts, the taxes on the efficient plans are zero.

Our first implementation result ${ }^{5}$ establishes that efficient allocations can always be implemented using fiat money, by introducing non-lump-sum taxes that correct for the long-term gap in the benefits and costs of saving plans. The tax schedule can be required to be non-distortionary (in the sense that Euler and transversality conditions are just as they were in an economy without taxes), or equivalently, that when applied in a context of impatience such taxes would make money supply go to zero.

While being non-lump-sum, taxes are impersonal and can be chosen to be levied upon the disposal of the assets, taxing the benefit from disposing of money balances net of the cost of carrying on cash up to then, when that difference happens to be positive ${ }^{6}$. Taxes have therefore a flavour of a capital gains tax.

We illustrate our implementation result with examples for preferences in the two cases described in 1.2. Moreover, we give also an example (Example 3) of an economy where there is an inefficient equilibrium with sequential budget constraints when the taxes that we propose are absent. In the $\mathrm{AD}$ equilibrium, the infimum of the consumption plans is not attained in finite time, but in the inefficient sequential outcome that infimum is attained at infinitely many dates, different for the two consumers. At the inefficient consumption plans, consumers' utility functions have some supergradients without pure charges and this allows for the consumers' problems to have finite optima under the plain no-shortsales constraint on money. In other words, there are no asymptotic gains from dishoarding under such supergradients and, therefore, equilibria under sequential budget constraints exist in the absence of our proposed taxes. However, for such supergradients, at dates where the infimum is attained, the left and the right marginal utilities do not coincide and no-short-sales constraints have shadow values, which implies that the marginal rates

\footnotetext{
${ }^{5}$ See our Theorem 2.

${ }^{6}$ This means that the accumulated taxes up to any moment in time (when the asset might be disposed of) are always non-negative, but the tax at each date might be negative, that is, allow for tax rebates.
} 
of inter-temporal substitution will not be the same for the two consumers.

\subsection{Why money?}

Choosing fiat money as the implementing asset has an important advantage. If AD net trades would seem to require negative cash balances at some dates, we can easily replace such unduly short positions by increasing the initial money holdings adequately. In an implementation with Lucas trees, such adjustment in initial holdings would have to be combined with a reduction in the commodity endowments of the sequential economy so that there would be no change in $\mathrm{AD}$ endowments (the sum of sequential endowments and returns from initial holdings of the tree). However, there is a bound on such substitution, as sequential endowments must remain non-negative.

Taking fiat money to be the implementing asset also allows for interesting comparisons with what the literature says. The reserve role that money plays in our context of lack of impatience is clearly novel and differs from other arguments for that role. Nevertheless, one can not help recalling the idea put forward by Friedman $(1953,1969)$ that consumers should not economize unnecessarily on money balances as these holdings are "a reserve against future emergencies". In our context, such future emergencies occur at distant dates but the lack of impatience makes them relevant. It resumes also Bewley's (1980) idea that the "device to give money a value is infinite horizon (together with the need for insurance)", but we take a step forward and take into account the limiting hedging role of money for non-impatient agents.

If, for any consumer and any date, marginal utilities are well defined at each date ${ }^{7}$ and consumption is positive at each date, then Friedman's zero nominal interest rate rule holds, that is, deflation must occur, at least at infinitely many dates (as we point out in Remark 1(ii), Section 3.2). In such deflationary context, a tax on gains on money dishoarding would

\footnotetext{
${ }^{7}$ This holds for the two classes of preferences mentioned in 1.2 when the relevant infimum is not attained in finite time.
} 
be the equivalent for money to what capital gains taxes are for all other assets. Actually, foreign currency balances tend to be subject to such taxes, like any other non-monetary asset. We are taking a step forward, suggesting that all money balances should be, in order for efficiency to be attained in a deflationary context where agents are wary and prone to dishoard in the long run.

\subsection{Non-vanishing money supply}

What can be said about the asymptotic money supply? Do taxes end up driving money holdings to zero, for all agents? We show that this does not have to be the case.

When some agents are non-impatient, a multiplicity of equilibrium money balances plans occurs, with different limiting supplies of money. Interestingly, there may exist one monetary equilibrium with non-vanishing money supply.

Such persistent hedging role of money is reminiscent of what Samuelson (1958) established for the overlapping generations model, which seemed until now incompatible with immortal agents consuming at every date. For impatient agents, Bewley (1980, 1983) showed that a non-vanishing money supply, together with interior consumption, had to be inefficient. Levine $(1986,1988,1989)$ confirmed this under Inada's condition but observed that efficiency might prevail under non-interior consumption ${ }^{8}$. Frequent corner solutions entail frequent gaps between prices and marginal utilities that are somehow akin to gaps that we find in this paper for the valuation of consumption at infinity.

A non-vanishing money supply occurs when for some non-impatient agent the personal appraisal of the loss from reverting the optimal savings plan is higher than the AD price of that plan (see our Theorem 3).

Such gap is a consequence of the non-differentiability of wary preferences at nonconverging net trades, due to the freedom in the choice of the generalized limit for the pure charge of the supergradient. The non-differentiability implies that, for a wary con-

\footnotetext{
${ }^{8}$ See also Woodford (1990), Kehoe et al. (1992) and Pascoa et al. (2010).
} 
sumer, the marginal loss from reverting a savings plan is greater than the marginal gain from intensifying that savings strategy ${ }^{9}$. The AD price will value the savings plan somewhere in between the latter and former. The limiting money supply turns out to be the sum, over all consumers, of how much the personal marginal losses from reverting the savings plans actually exceed the AD pricing of those plans.

We illustrate a positive asymptotic money supply (Example 2), both for the precautionary and the habit persistent preferences, by allowing for frequent endowment shocks. If for some consumer, the infimum of lifetime utilities (for the former) or the infimum of average utilities up to each point in time (for the latter) is not attained in finite time, then such consumer has a marginal loss from reverting savings that is above the $\mathrm{AD}$ price of those savings. The mismatch of personal and $\mathrm{AD}$ prices requires agents to hold on to cash forever in order to hedge frequent endowment shocks. In other words, the bubble is not entirely sold due to such mismatch.

While our main implementation result is, in many contexts ${ }^{10}$, in line with a proposition by Friedman $(1953,1969)$ that the wasteful economizing of cash should be avoided by deflation or by providing money with a real rate of interest, our analysis of how money supply evolves is not so much in line with the stronger recommendation of a steady contraction of the money supply, which has been often associated with that proposition by Friedman.

The rest of the paper is organized as follows. Endogenous discounting is described and related to wariness in section 2 (illustrating with the precautionary and the habit persistence examples). Section 3 presents the deterministic sequential monetary model and Section 4 states and illustrates the results on efficient monetary equilibrium. The concluding remarks in Section 5 compare our work with the implementation using non-

\footnotetext{
${ }^{9}$ This asymmetry is reminiscent of the asymmetrical attitude towards losses and gains proposed in prospect theory.

${ }^{10}$ More precisely, under interior consumption and well defined marginal utilities date by date, for all consumers.
} 
monetary assets, as in Araujo et al. (2011) which just dealt with the $\epsilon$-contamination case and also differs from our work by having used portfolio constraints rather than taxes. Finally, section 5 points out also that the preferences that we contemplate allow for timeinconsistency but are actually time-consistent in $\mathrm{AD}$ equilibria with price pure charges. All proofs are presented in the Appendix.

\section{Endogenous Discounting}

We assume that infinite-lived consumers have a collection of possible discount factor sequences and, not being sure which one to pick, end up choosing for each consumption plan the discount factor sequence that gives the lowest sum for the series of discounted utilities. Such attitude is a form of endogenous discounting already addressed by Geoffard (1996) and which has received significant attention in the recent literature on the (mis)specification of macro models (see Hansen and Sargent (2001, 2008)).

To be quite general, preferences are described by

$$
U(x)=\inf _{\delta \in C} \sum_{t=1}^{\infty} \delta_{t} u\left(x_{t}\right),
$$

where the set $C$ is some subset of $\ell_{+}^{1} \cap B_{1}(0)$, where $B_{1}(0)$ is the unit ball of $\ell^{1}$.

The preferences defined above have an analogy with the ambiguity aversion attitude in a context of uncertainty, the maxmin model (see Gilboa and Schmeidler (1989)). This model contemplates a functional which is the minimum of the expected utilities over a collection of beliefs described by finitely additive set functions, that is, given a general set $X$ of possible events or states (in our context $X=\mathbb{N}$ ),

$$
\mathbf{V}(\mathbf{x})=\min _{\eta \in \tilde{C}} \int_{X} u \circ x d \eta
$$

where $\tilde{C}$ is a convex and weak* ${ }^{*}$ closed subset of $b a$ (the referred papers discussed axiomatically this representation ${ }^{11}$. The minimal integral over beliefs represents a precautionary

\footnotetext{
${ }^{11}$ See Dunford and Schwartz (1958), ch. III.2, for the definition of integral with respect to a charge $\eta$.
} 
or pessimistic behavior. The minimization solution $\eta^{*}$ puts more weight on sets where $u$ attains its lowest values. To see that Equation (1) can be reformulated in terms of Equation (2), for an appropriate (not any) choice for the set $\tilde{C}$, we take $\tilde{C}$ to be the closure in the weak* topology of the convex hull of $C$ and we get $U(x)=V(x)^{12}$.

\subsection{Endogenous discounting and wariness}

As in Araujo et al. (2011), we say that infinite-lived consumers are wary when they neglect distant gains but not the losses at far away dates. This consists in being upper but not lower semi-impatient. Let us formalize these concepts, presuming monotonicity of preferences $\succeq^{i}$ over sequences of consumption of the single good. For any sequence $v \in \ell^{\infty}$ we denote by $v(n)$ the sequence such that $v(n)_{t}=v_{t}$ for $t \geq n$ and $v(n)_{t}=0$ otherwise.

Consumer $i$ is said to be upper semi-impatient at a bundle $x$ if $x \succ^{i} y$ implies, for any $z \in \ell_{+}^{\infty}$, that $x \succ^{i} y+z(n)$ for $n$ large enough. Consumer $i$ fails to be lower semi-impatient at a bundle $x$ if there exists $y$ for which $y \succ^{i} x$ but $x \succeq^{i} y-y(n), \forall n$. Losses beyond date $n$ reverse the preference ordering, no matter how large $n$ is.

These concepts can be formulated in terms of the Mackey topology on $\ell^{\infty}$, the finest topology on $\ell^{\infty}$ for which the dual is $\ell^{1}$. For norm-continuous preferences $\succeq^{i}$, upper (lower) semi-impatience at $x$ consists in the Mackey upper (lower) semi-continuity of $\succeq^{i}$ at $x$. A consumer whose preferences are norm continuous and Mackey upper semi-continuous, is wary at $x \in \ell_{+}^{\infty}$ if the preferences are not Mackey lower semi-continuous at $x$. If this property holds on the norm interior of $\ell_{+}^{\infty}$, the consumer is said to be wary. Even though the economy is deterministic, we can interpret the consumer's cautious attitude towards distant losses as reflecting some wariness that is actually related, as we saw, to not being sure how such losses should be discounted.

We present next the most that one can say without imposing more structure on the set

\footnotetext{
${ }^{12}$ This follows from the compacity of the set $\tilde{C}$ in the weak* topology and the continuity of the functional $x \mapsto \int_{X} u \circ x d \eta$ in the weak* topology.
} 
$C$ of possible discount factors.

Lemma 1 (Aversion to ambiguity and wariness). Under aversion to the ambiguity in the discount factors, that is, when preferences are given by Equation (1), preferences are Mackey upper semi-continuous but may fail to be Mackey lower semi-continuous.

This is a consequence of the fact that the lower envelope of a family of upper semicontinuous functions (on any topological space) is still upper semi-continuous. Other results can be obtained by specifying the set $C^{13}$.

Assumption $\mathbf{H}$ : preferences are described by an utility function given by Equation (1) where (i) the utility index $u: \mathbb{R}_{+} \rightarrow \mathbb{R}$ is concave and strictly increasing and (ii) $C \subset\left\{\delta \in \ell_{+}^{1} \cap B_{1}(0): \delta_{t} \geq \varepsilon_{t}\right\}$ for some sequence $\varepsilon$ such that $\varepsilon_{t}>0$ for each $t$.

Since $\left\{\delta_{t}\right\}_{\delta \in C}$ is bounded away from zero at each $t$, if $u$ is strictly increasing, so will $U$ (that is, $U(x)>U\left(x^{\prime}\right)$ whenever $\left.x>x^{\prime}\right)^{14}$.

The following property holds under $\mathbf{H}$ :

Condition C1: for each agent, preferences are representable by a utility function $U^{i}$ that is concave, norm continuous, Mackey upper semi-continuous and such that $U^{i}(x)>U^{i}\left(x^{\prime}\right)$ whenever $x>x^{\prime}$.

Note that there are several preferences that satisfy Condition C1 but not assumption $\mathbf{H}$ (do not have a representation satisfying Equation (1)).

We start by examining the case of a set $C$ generated by a capacity, that is, a function $\nu: 2^{\mathbb{N}} \rightarrow \mathbb{R}$ such that $\nu(\emptyset)=0$ and $\nu(A) \leq \nu(B)$ whenever $A \subseteq B$. A capacity $\nu$ is convex when $\nu(A \cup B)+\nu(A \cap B) \geq \nu(A)+\nu(B) \forall A, B \subset \mathbb{N}$. We normalize $\nu(\mathbb{N})=1$. The set $\operatorname{core}(\nu)$ is defined as $\{\eta \in b a: \eta \geq \nu, \eta(\mathbb{N})=1\}$. When $C=\operatorname{core}(\nu)$ for a convex capacity $\nu$, the utility function is a Choquet integral (see Schmeidler (1989)) and more

\footnotetext{
${ }^{13}$ Note that Equation (1) allows for but does not imply Mackey lower semi-discontinuity. The choice of deflators that the set $\mathrm{C}$ allows for may even pertain to just a finite subset of dates, in which case time-separability would fail just in that subset and Mackey lower semi-continuity would hold.

${ }^{14}$ In $\ell^{\infty}, x>y$ means that $x_{t} \geq y_{t}$ for all $t$ and $x \neq y, x \gg y$ means that $x_{t}>y_{t}$ for all $t$, and $x \ggg y$ means that there is $\varepsilon>0$ such that $x_{t}+\varepsilon \geq y_{t}$ for all $t$.
} 
can be said about the absence of Mackey lsc. A capacity $\nu$ is said to be continuous at certainty if, for any sequence $\left(A_{n}\right) \subset 2^{\mathbb{N}}$ such that each $A_{n} \subset A_{n+1} \subset \mathbb{N}$ and $\cup_{n} A_{n}=\mathbb{N}$, we have $\lim \nu\left(A_{n}\right)=\nu(\mathbb{N})$. Now, $U$ is Mackey lsc if and only if the capacity is continuous at certainty (by Theorem 2.1 in Araujo (1985)). The discontinuity at certainty can be interpreted as if there were a missing state. In Araujo et al. (2011), the focus was on a well-known example of a convex capacity, which we recall next.

\section{Example 1}

Given a probability measure $\mu$ and $\epsilon \in[0,1)$, let $\nu_{\epsilon}$ be the convex capacity defined by $\nu_{\epsilon}(A)=(1-\epsilon) \mu(A)$ for $A \varsubsetneqq \mathbb{N}$ and $\nu_{\epsilon}(\mathbb{N})=1$. This is called the $\epsilon$-contamination capacity with respect to $\mu$. As shown by Dow and Werlang (1992) we can rewrite (2) as ${ }^{15}$

$$
U(x)=(1-\epsilon) \int_{\mathbb{N}} u \circ x d \mu+\epsilon \inf u \circ x
$$

Clearly, $\nu_{\epsilon}$ is discontinuous at certainty and, therefore, this utility represents wary preferences at some point ${ }^{16}$. Actually, for some $(\zeta, \beta)$ proportional to $((1-\epsilon) \mu, \epsilon)$, the utility can be rewritten (up to a scalar multiple) as

$$
U(x)=\sum_{t=1}^{\infty} \zeta_{t} u\left(x_{t}\right)+\beta \inf _{t \geq 1} u\left(x_{t}\right)
$$

Under (4) time-consistency may not hold, but it does if the infimum of consumption is not attained. This will be the case when we resume Example 1.

We introduce another example of wary preferences where the concern about the infimum is weaker than in Example 1. The non-additively separable part of the utility function will be related to the Polya index introduced by Marinacci (1998) to describe patience. To do so, we define a countable set of priors in $\ell_{+}^{1}$ which induces a smaller set of priors in $b a$ than the one obtained from the $\epsilon$-contamination capacity.

\section{Example 2}

\footnotetext{
${ }^{15}$ In this case, the minimum over normalized dominating charges coincides with the infimum over dominating probabilities: $U(x)=\inf \left\{\int_{\mathbb{N}} u \circ x d \eta: \eta \in c a \cap \operatorname{core}\left(\nu_{\epsilon}\right)\right\}$.

${ }^{16}$ Actually, wariness holds at every $x \ggg 0$ as the lower contour set of $x$ is not Mackey closed.
} 
The agent set of beliefs $C$ is now defined by $C:=\operatorname{core}\left(\nu_{\epsilon}\right) \cap \hat{C}$ where $\hat{C}$ is the closed convex hull of $\left\{\left(\delta_{m}\right)_{m \in \mathbb{N}}: \delta_{m}(t)=\zeta_{t}+\beta^{i} / m\right.$ for $1 \leq t \leq m, \delta_{m}(t)=\zeta_{t}$ elsewhere $\}$ in the weak* topology of $b a$. Then, the utility function in (1) becomes, by multiplying by a suitable scalar,

$$
U(x)=\sum_{t} \zeta_{t} u\left(x_{t}\right)+\beta \inf _{t}\left(\frac{1}{t} \sum_{k=1}^{t} u\left(x_{k}\right)\right) .
$$

Marinacci (1998) came up with a notion of complete patience using the Polya index, $\lim _{t}\left(\frac{1}{t} \sum_{k=1}^{t} u\left(x_{k}\right)\right)$, which has a similarity with the last term in Equation $(5)^{17}$, although the replacement of limit by the infimum implies that the agent now cares about small consumption in the first dates and, therefore, the agent has some degree of impatience. The time separable component of $U$ also enhances the level of impatience, but some patience prevails, as the agent is worried about mean losses, for means computed up to any distant date. This is a form of habit persistence.

\subsection{Wariness and Arrow Debreu Equilibrium}

Suppose there are $I$ infinitely lived consumers, who are endowed with quantities $\omega_{t}^{i} \geq 0$ of a single commodity at the countably many dates.

Wariness impacts on the nature of the supporting prices of efficient allocations. An Arrow-Debreu equilibrium (AD) is defined as a pair $(x, \pi)$ such that $x=\left(x^{1}, \ldots, x^{I}\right)$ is a feasible allocation, $\pi$ a linear functional on $\ell^{\infty}$ and, for each $i, x^{i}$ maximizes $U^{i}$ in the budget set $\left\{a \in \ell_{+}^{\infty}: \pi\left(a-\omega^{i}\right) \leq 0\right\}$. The natural environment where to look for prices is the norm dual of $\ell^{\infty}$. This is the space $b a$, of bounded finitely additive set functions, also called charges, on $\mathbb{N}$, equipped with the total variation norm (given $b \in b a$, its norm

\footnotetext{
${ }^{17}$ Note that Marinacci's (1998) complete patience preference cannot be obtained as described in Equation (1), but it can be obtained from Equation (2) for a set $\tilde{C}$ that only contains the Polya index (a set that cannot be generated as a convex hull of elements in $\left.\ell_{+}^{1} \cap B_{1}(0)\right)$. In any case, this preference is neither Mackey usc nor lsc.
} 
is $\|b\|=|b|(\mathbb{N}))$. Let us rephrase a well-known result by Bewley (1972).

Proposition 1. If $\boldsymbol{C} 1$ holds and $\sum_{i} \omega^{i} \ggg 0$, there exists an $A D$ equilibrium $(x, \pi)$, with the price $\pi$ in ba. Some consumer being wary at $x^{i}$ is a necessary condition for $A D$ equilibrium prices to be outside of $\ell^{1}$.

Notice that $b a$ contains strictly $\ell^{1}$, the space of absolutely summable sequences, since each $y \in \ell^{1}$ induces an element $\mu$ in the space $c a$ of countably additive set functions on $\mathbb{N}$ (by setting $\left.\mu(\{t\})=y_{t}\right)$.

Comment on Example 1 and the existence of AD equilibrium: Araujo (1985) showed that the Mackey topology is the finest topology of continuity in order for AD equilibrium to exist (with prices in $b a$ ), if no further assumptions are imposed, except for the convexity of preferences. This result was illustrated by claiming that for $U(x)=\inf _{t} u\left(x_{t}\right)$ equilibrium does not exist. However, when monotonicity with respect to increments at finitely many dates is added (which is satisfied by Equation (4)) AD equilibrium (with prices in $b a$ ) exists, in spite of the failure of Mackey lower semi-continuity, as Bewley (1972) established. The other case of pessimistic preferences, with $U(x)=\liminf _{t} u\left(x_{t}\right)$, mentioned in Araujo (1985) has the drawback that the upper Mackey semi-continuity does not hold and, even if a series of discounted utilities would be added, $\mathrm{AD}$ equilibrium might not exist.

\subsection{Characterization of AD Equilibrium Prices}

Let us characterize the $\mathrm{AD}$ equilibria whose prices fail to be in $\ell^{1}$. We start by examining how do supporting prices look like.

By the Yosida-Hewitt Theorem, any $\pi \in b a$ can be decomposed uniquely in the form $\pi=\mu+\nu$ where $\mu \in c a$ whereas $\nu$ is a pure charge. For any finite subset $B$ of $\mathbb{N}$, if $\nu$ is a positive pure charge, then $\nu(B)=0$. Denote by $p c h$ the set of pure charges on $\left(\mathbb{N}, 2^{\mathbb{N}}\right)$. Let us see characterize the pure charge components of a supporting price.

Recall that $T \in b a$ is a supporting price for an allocation $\left(x^{i}\right)_{i}$ if $U^{i}(z) \geq U^{i}\left(x^{i}\right)$ implies that $T\left(z-x^{i}\right) \geq 0$ for any $i$. A supporting price is, up to a positive scalar multiple, a 
supergradient ${ }^{18}$ of $U^{i}$ at $x^{i}$. Denote by $\partial U^{i}(x)$ the set of supergradients of $U^{i}$ at $x$. For any $v \in \ell^{\infty}$ we denote by $\delta^{+} U^{i}(x ; v)$ and $\delta^{-} U^{i}(x ; v)$ the right and left derivatives of $U^{i}$ at $x$ along the $v$-direction ${ }^{19}$. Let $\mathbb{Z}(n)$ be the real sequence whose first $n$ terms are zero and the remaining terms are equal to 1.

Lemma 2. If $\nu \in p c h_{+}$, then $\nu(x) /\|\nu\|_{b a} \in[\liminf x, \lim \sup x], \forall x \in \ell^{\infty}$. If for some $\mu \in c a, \mu+\nu \in \partial U^{i}(x)$, then $\|\nu\|_{b a} \in\left[\lim _{n} \delta^{+} U^{i}(x ; \mathbb{Z}(n)), \lim _{n} \delta^{-} U^{i}(x ; \mathbb{Z}(n))\right]$

We use the notation $L I M$ to represent a linear functional taking on each $x \in \ell^{\infty}$ a value in $[\lim \inf x, \lim \sup x]$. Notice the real indeterminacy in $A D$ equilibrium resulting from the choice of $L I M$ for the pure charge $\nu$ in the support price (if we pick another $L I M$, the $\mathrm{AD}$ budget equation will not hold for the same bundle $x^{i}$, except when the supergradients at $x^{i}$ have also multiple $c a$ components). Wariness is necessary for the occurrence of pure charges supporting interior bundles:

Lemma 3. Under $\boldsymbol{C 1}$, if $U^{i}$ is Mackey continuous, then $\partial U^{i}(x) \subset \ell^{1}$ for $x \ggg 0$.

Let us go back to Examples 1 and 2 and characterize the supergradients of the utility functions in these examples. We denote the infimum of a bundle $x$ by $\underline{x}$. In Example 1 , any supergradient $T$ of $U$ at $x$ must be of the form ${ }^{20} T(a)=\sum_{t+1}^{\infty} u^{\prime}\left(x_{t}\right)\left(\zeta_{t}+\gamma_{t} \beta\right) a_{t}+$ $\sigma \beta u^{\prime}(\underline{x}) \operatorname{LIM}^{T}(a)$, for any $a \in \ell^{\infty}$, where (i) $\gamma_{t} \geq 0$, (ii) $\gamma_{t}=0$, if $x_{t}>\underline{x}$, (iii) $\sigma \geq 0$ is zero when $\underline{x}$ is not a cluster point of $x$ and (iv) $\sum_{t=1}^{\infty} \gamma_{t}+\sigma=1$. That is, there is a supergradient with a pure charge only if $\underline{x}$ is a cluster point of $x$ and all supergradients will have pure charges if $\underline{x}$ is not attained.

In Example 2, for any $\left(x_{t}\right)_{t}$ such that the infimum of $\left(\frac{1}{t} \sum_{k=1}^{t} u\left(x_{k}\right)\right)_{t}$ is not attained, the supporting prices have the following form $\pi(c)=\sum_{t=0}^{\infty} \zeta_{t}(u)^{\prime}\left(x_{t}\right) c_{t}+\beta^{i} \operatorname{LIM}(\phi(c))$ where $\phi: \ell_{+}^{\infty} \rightarrow \ell_{+}^{\infty}$ such that $\phi(c)_{t}=\frac{1}{t} \sum_{k=1}^{t} c_{k}$. The presence of a pure charge is due to the fact that the series of marginal utilities does not converge uniformly on the countably many

\footnotetext{
${ }^{18} T \in b a$ is a supergradient of $U^{i}$ at $x$ if $U^{i}(x+h)-U^{i}(x) \leq T h$ for any $h \in \ell^{\infty}$.

${ }^{19} \delta^{+} U(x ; v)=\lim _{h \downarrow 0} \frac{U(x+h v)-U(x)}{h}$ and $\left.\delta^{-} U(x ; v)\right)$ is defined with $h \uparrow 0$ instead.

${ }^{20}$ For a proof see Araujo et al. (2011).
} 
deflators $\delta_{m}$ that induce the set $C$ of discount beliefs in this example. This leads us to formulate a general result, presented in the next subsection.

\subsection{Pure charges and the non-uniform convergence of marginal utility series}

In general what can be said about the occurrence of supporting prices outside of $\ell^{1}$ for wary preferences generated by endogenous discounting of the form of Equation (1)? We examine what happens with the family $\left\{\sum_{t} \delta_{t} u^{\prime}\left(x_{t}\right)\right\}_{\delta \in C}$ of marginal utilities, for deflators $\delta$ in $C$.

Theorem 1. (Pure charges and the non-uniform convergence of marginal utilities) For the utility function defined by Equation (1), suppose $C \subseteq \ell_{++}^{1}$ and take $\left(x_{t}\right)_{t \in \mathbb{N}} \ggg$ 0. If the series of marginal utilities at $\left(x_{t}\right)_{t \in \mathbb{N}}$ converges uniformly on the set $C$, in the sense that $\lim _{t} \sup _{\delta \in C}\left\{\sum_{s \geq t} \delta_{s} u^{\prime}\left(x_{s}\right)\right\}=0$, then there is no pure charge in any supergradient of $U$ at $x$.

\section{A Sequential Economy with Fiat Money}

\subsection{Money and Taxes}

The set of trading dates is $\mathbb{N} \equiv\{1,2, \ldots\}$. Before the initial date, the government allocates non-negative initial holdings $y_{0}^{i} \geq 0$ of money to each consumer and then, at each trading date $t$, money holdings $y_{t}^{i}$ may be taxed. The taxes levied at each date, denoted by $\tau_{t}\left(y^{i}\right)$, may depend on the whole individual plan $y^{i}$ of money holdings, but through an impersonal tax schedule $\tau_{t}($.$) .$

The consumption good is the numeraire and we denote by $q=\left(q_{t}\right)_{t \in \mathbb{N}}$ the sequence of prices of money. Every consumer $i$ faces at each date $t \in \mathbb{N}$, the following constraints:

$$
y_{t} \geq 0
$$




$$
x_{t}-\omega_{t}^{i} \leq q_{t}\left(y_{t-1}-y_{t}-\tau_{t}(y)\right)
$$

Observe that $\tau_{t}(y)$ just has an impact at date $t$ when $q_{t}>0$.

Let us denote by $B\left(q, y_{0}^{i}, \omega^{i}, \tau\right)$ the set of couples $(x, y) \in \ell_{+}^{\infty} \times \mathbb{R}_{+}^{\infty}$ of consumption and money holdings plans satisfying constraints (6) and (7). The goal of agent $i$ is to maximize $U^{i}$ under $B\left(q, y_{0}^{i}, \omega^{i}, \tau\right)$. We denote the set $\{1, \ldots, I\}$ of agents by $\mathcal{I}$.

The initial money supply $M_{0}$ is given, equal to $\sum_{i=1}^{I} y_{0}^{i}$ and assumed to be positive. However, at each trading date, the money supply $M_{t}$ is endogenous, satisfying

$$
M_{t}\left(y^{1}, \ldots, y^{I}\right)=M_{t-1}\left(y^{1}, \ldots, y^{I}\right)-\sum_{i=1}^{I} \tau_{t}\left(y^{i}\right)=\sum_{i=1}^{I}\left(y_{0}^{i}-\sum_{s=1}^{t} \tau_{s}\left(y^{i}\right)\right)
$$

Definition 1. $\left(q,\left(x^{i}, y^{i}\right)_{i \in \mathcal{I}}\right) \in \mathbb{R}_{+}^{\infty} \times\left(\ell_{+}^{\infty} \times \mathbb{R}_{+}^{\infty}\right)^{I}$ is an equilibrium for the economy with initial money holdings $\left(y_{0}^{1}, \ldots, y_{0}^{I}\right)$ and a tax policy $\tau$ if $(\mathrm{a})\left(x^{i}, y^{i}\right) \in \operatorname{argmax}\left\{U^{i}(x):(x, y) \in\right.$ $\left.B\left(q, y_{0}^{i}, \omega^{i}, \tau\right)\right\}$; (b) $\sum_{i=1}^{I}\left(x^{i}-\omega^{i}\right)=0$; (c) $M_{t}\left(y^{1}, \ldots, y^{I}\right)=\sum_{i=1}^{I} y_{t}^{i} \forall t \in \mathbb{N}$.

Definition 2. An equilibrium $\left(q,\left(x^{i}, y^{i}\right)_{i \in \mathcal{I}}\right)$ is a monetary equilibrium if $q \neq 0$.

If $q_{t_{0}}>0$ for some date $t_{0}$, it will be true by non-arbitrage that $q_{t}>0 \forall t$. Note that, under $\mathrm{C} 1,(7)$ holds as equality, which summed over $i$, make $(b)$ imply $(c)$.

\subsection{Sequential equilibrium and improvement opportunities}

Observe first that in the absence of any taxes, sequential budget constraints are as follows

$$
x_{t}-\omega_{t}^{i} \leq q_{t}\left(z_{t-1}-z_{t}\right) \quad \forall t \in \mathbb{N},
$$

A very useful sufficient condition for individual optimality is given as follows. Let $x(z, i)$ be defined by $x_{t}(z, i)=\omega_{t}^{i}+q_{t}\left(z_{t-1}-z_{t}\right)$.

Proposition 2. Let $z^{i}$ be a portfolio plan satisfying Equations (6) and (8) and let $x^{i}=$ $x\left(z^{i}, i\right)$. (i) Suppose there exists $T \in \partial U\left(x^{i}\right)$ with $T=\mu+\nu, \mu \in \ell_{+}^{1}$ and $\nu \in \mathrm{pch}_{+}$such that, for every $t$,

$$
\mu_{t} q_{t} \geq \mu_{t+1} q_{t+1} \quad\left(\mu_{t} q_{t}-\mu_{t+1} q_{t+1}\right) z_{t}^{i}=0
$$


and

$$
\lim \mu_{t} q_{t} z_{t}^{i}=\nu\left(x^{i}-\omega^{i}\right)
$$

(ii) Suppose also that every feasible portfolio z satisfies the condition

$$
\lim _{t} \mu_{t} q_{t} z_{t} \geq \nu\left(x(z, i)-\omega^{i}\right)
$$

Then $z^{i}$ is an optimal solution for the problem with constraints (6) and (8).

Proof. Given a feasible portfolio $z, U(x(z, i))-U\left(x^{i}\right) \leq T\left(x(z, i)-x^{i}\right)=T(x(z, i)-$ $\left.\omega^{i}\right)+T\left(\omega^{i}-x^{i}\right)$. Moreover, $\mu\left(x(z, i)-\omega^{i}\right)=\sum_{t=1}^{\infty} \mu_{t} q_{t}\left(z_{t-1}-z_{t}\right)$. By (9) and $z \geq 0$, $\mu\left(x(z, i)-\omega^{i}\right) \leq \mu_{1} q_{1} z_{0}-\lim _{t} \mu_{t} q_{t} z_{t}$. Also, $\mu\left(x^{i}-\omega^{i}\right)=\mu_{1} q_{1} z_{0}-\lim _{t} \mu_{t} q_{t} z_{t}^{i}$. Now by $(10)$, $U(x(z, i))-U\left(x^{i}\right) \leq \nu(x(z, i)-\omega)-\lim _{t} \mu_{t} q_{t} z_{t}$. Now, by $(11), U(x(z, i))-U\left(x^{i}\right) \leq 0$.

\section{Remark 1: Comparison with necessary conditions}

(i) Given $q$ and $y_{0}^{i}$, a plan $y^{i}$ is optimal for agent $i$ only if there is some supergradient $T \in \partial U^{i}\left(x^{i}\right)$ whose $c a$ component $\mu^{i}$ satisfies Euler conditions (9).

(ii) Suppose that an $\mathrm{AD}$ equilibrium $\left(x, p+\nu^{A D}\right)$ is such that, for all $i$, (iia) $x^{i} \gg 0$ and (iib) marginal utilities $\mu_{t}^{i}$, at each $t$, are well defined ${ }^{21}$. From (iib) we get that all supergradients of $U^{i}$ at $x^{i}$ have the same $c a$ component $\mu^{i}$. From (iia) we get that $\mu^{i}$ is collinear with $p$ for all $i$. It follows that condition (9) must hold with no slack (otherwise agents holding money would have marginal rates of substitution different from those of other agents).

Under assumptions (iia) and (iib), any monetary equilibrium implementing the efficient allocation $x$ is such that the inflation rate $\left(\frac{1 / q_{t+1}}{1 / q_{t}}-1\right)$ is equal to the consumers' rate of rate of time preference $\left(\mu_{t+1}^{i} / \mu_{t}^{i}\right)$ minus one. Now, as $\mu^{i} \in \ell^{1}$, this implies

\footnotetext{
${ }^{21}$ That is, the directional derivative of $U^{i}$ exists at $x^{i}$. This is not a technical assumption, it is instead a property that depends on the asymptotic behavior of the consumption sequence, as was illustrated is Example 1.
} 
3 A SEQUENTIAL ECONOMY WITH FIAT MONEY

deflation, at least at infinitely many dates (actually, always beyond some date when consumption converges to some positive level). Moreover, under (iia) and (iib), Friedman's weak rule, prescribing a zero nominal interest rate, holds, since there is no room in the Euler equations to replace $q_{t+1}$ by $q_{t+1}(1+i)$ with a positive interest rate $i$.

(iii) Condition (10) is related to another necessary condition, the transversality condition. Suppose again Condition (iib) of item (ii) and let $P\left(x^{i}\right)=\partial U^{i}\left(x^{i}\right)-\mu^{i}$ be the set of pure charges of all supergradients. Suppose consumer $i$ moves in the direction $z^{i}(n)$, that is, replaces $z_{t}^{i}$ by $(1+h) z_{t}^{i}$ from some date $n$ onward. The associated direction of changes in consumption is $c\left(z^{i}(n)\right)_{t}=0$ if $t<n, c\left(z^{i}(n)\right)_{n}=-q_{n} z_{n}^{i}$ and $c\left(z^{i}(n)\right)_{t}=q_{t}\left(z_{t-1}^{i}-z_{t}^{i}\right)=x_{t}-\omega_{t}^{i}$ if $t>n$. Now, $\mu^{i}\left(c\left(z^{i}(n)\right)\right)=-\mu_{n}^{i} q_{n} z_{n}^{i}+$ $\sum_{t>n} \mu_{t}^{i} q_{t}\left(z_{t-1}^{i}-z_{t}^{i}\right)$ which by (9) reduces to $-\lim \mu_{t}^{i} q_{t} z_{t}^{i}$. Hence, there is no utility gain by changing portfolios in the $z^{i}(n)$ direction along the right (for $h>0$ ) only if (iiia) $\delta^{+} U^{i}\left(x^{i}, c\left(y^{i}(n)\right) \equiv-\lim \mu_{t}^{i} q_{t} z_{t}^{i}+\min _{\nu^{i} \in P\left(x^{i}\right)} \nu^{i}\left(x^{i}-\omega^{i}\right) \leq 0\right.$ and no utility gain by moving along $z^{i}(n)$ on the left (for $h<0$ ) only if (iiib) $\delta^{-} U^{i}\left(x^{i}, c\left(y^{i}(n)\right) \equiv\right.$ $-\lim \mu_{t}^{i} q_{t} z_{t}^{i}+\max _{\nu^{i} \in P\left(x^{i}\right)} \nu^{i}\left(x^{i}-\omega^{i}\right) \geq 0$ (irrespective of the presence of constraints of the form (11)). Hence, (10) must hold for some supergradient.

Notice also that transversality conditions do not imply that efficient individual money balances must go to zero. The latter would hold if for the pure charges $\nu^{i}$ attaining the maximum in (iiib) we would have $\sum_{i} \nu^{i}\left(x^{i}-\omega^{i}\right)=0$, which is the case when all net trades converge $\left(\right.$ as $\left.\nu^{i}\left(x^{i}-\omega^{i}\right)=\nu^{A D}\left(x^{i}-\omega^{i}\right)\right)$.

\section{Remark 2: On sufficiency}

When agents are not impatient, long-run improvement opportunities are not ruled out by no-short-sales constraints. In fact, take any non-negative real sequence $z$ and let $x \equiv$ $x(z, i)$. Suppose consumer $i$ replaces $z_{t}^{i}$ by $z_{t}^{i}+h z_{t}$, with $h>0$, from some date $n$ onward. Analogously, to Remark 1 (iii), as we move on the right along this direction, we hoard more at date $n$ and at subsequent dates for which $\omega_{t}^{i}>x_{t}$, in order to consume more at subsequent dates where $\omega_{t}^{i}<x_{t}$. There would be a utility gain by doing so if the right 
derivative were positive. Now, the right derivative is the minimum of the values that all supergradients may take at this direction and, therefore, such improvement strategy is ruled out if there is a supergradient $T=\mu+\nu$ for which $T(c(z(n))) \leq 0$. Condition (11) implies the latter (and is equivalent to it in the absence of slacks in (9)).

Instead of restricting agents' choice sets to the portfolio plans satisfying (11), we introduce taxes that will discourage such improving strategies, more precisely, taxes that, for any portfolio plan, manage to eliminate the gap between the benefit from asymptotic dishoarding $\nu\left(x(z, i)-\omega^{i}\right)$ and the respective cost $-\mu(c(z(n)))$ of carrying on cash. Now, $-\mu(c(z(n)))=\mu_{n} q_{n} z_{n}+\sum_{t>n} \mu_{t} q_{t}\left(z_{t}-z_{t-1}\right) \geq \lim \mu_{t} q_{t} z_{t}$ (with a strict equality occurring only when there are slacks in (9)).

Consider the sequential implementation of an $\mathrm{AD}$ equilibrium $(x, \pi)$, for some endowments allocation $\omega$. The presence of a tax $\tau_{t}(y)$ levied at date $t$ upon a plan $y$ of money holdings implies that the funds that are actually put aside at each date are $z_{t}=y_{t}+\sum_{s \leq t} \tau_{s}(y)$. Taxes manage to close the gap between asymptotic dishoarding benefit and the cost of carrying on cash if (11) holds, for supergradients $\mu^{i}+\nu^{i}$, whose $c a$ component $\mu^{i}$ is collinear with the $c a$ component $p$ of the $\mathrm{AD}$ price $\pi$. The next section specifies taxes that have this property.

\section{Monetary Implementation of Efficient Allocations}

\subsection{Efficient Capital Taxation}

We impose the following assumption on taxes levied upon money holdings:

Condition C2: for any portfolio plan $y$ of any agent, taxes $\tau(y)$ are such that $(A)$ $\sum_{t=1}^{\infty} \tau_{s}(y) \geq \limsup q_{t}\left(y_{t-1}-y_{t}\right)-\lim y_{t},(B) \lim _{t} q_{t} \tau_{t}(y)=0$ if $x(y)$ is bounded and $(C)$ at any monetary equilibrium allocation $\left(y^{i}\right)_{i}$ the derivative of $\tau_{t}$ is null for any direction involving just changes in money balances at finitely many dates.

Item (A) requires taxes to close the gap between asymptotic dishoarding and the cost 
of carrying on cash. Item (B) implies that the limsup of the real value of cash disposals $\left(q_{t}\left(y_{t-1}-y_{t}\right)\right)$ is equal to the lim sup of the commodity net trade $\left(x(y)_{t}-\omega_{t}^{i}\right)$, for any money balances. Taxes being unaffected by changes in cash balances at finitely many dates is a property that is only required by item (C) to hold at equilibrium cash balances plans, as we will illustrate below.

\section{Theorem 2. (Efficient monetary equilibrium)}

Suppose preferences satisfy $\boldsymbol{C} \mathbf{1}$ and taxes satisfy $\boldsymbol{C} 2$. Let $(x, \pi)$ be an AD equilibrium for $\left(\omega^{i}\right)_{i}$ such that $x \gg 0$. Then, there exist initial money holdings $\left(y_{0}^{i}\right)_{i}$ so that $x$ can be implemented as the consumption allocation of a monetary equilibrium.

The proof in the Appendix contemplates all possible cases, but there is a case where the intuition behind the result is quite straightforward and is also what prevails in Examples 1 and 2 . When at $x^{i}$, the marginal utilities $\mu_{t}^{i}$ are well defined and the pure charges of all supergradients have the same norm $\alpha^{i}$, then (11) holds if (D) $\lim \mu_{t}^{i} q_{t} z_{t} \geq \alpha^{i} \lim \sup (x(z)-$ $\left.\omega^{i}\right)$. Now, $\mathrm{AD}$ prices $p+\alpha \operatorname{LIM}^{A D}$ can be normalized so that $\alpha=1$. Dividing both sides of the inequality by the AD Lagrange multiplier $\rho^{i}$ and making $q_{t}=1 / p_{t}$, we see that (D) becomes condition (A) in $\mathrm{C} 2$.

Now, the $\mathrm{AD}$ budget equation holds for $q_{t}=1 / p_{t}$ if $z_{0}^{i}=\lim _{t} z_{t}^{i}-\operatorname{LIM}^{A D}\left(x^{i}-\omega^{i}\right)$. Suppose we make $z_{0}^{i} \equiv \lim \sup \left(x^{i}-\omega^{i}\right)-\operatorname{LIM}^{A D}\left(x^{i}-\omega^{i}\right)$, then, $\lim z_{t}^{i}=\lim \sup \left(x^{i}-\omega^{i}\right)$ and, therefore, (10) holds. The implementation is done as long as the constructed $z^{i}$ is non-negative. Otherwise, initial money holdings should be adequately raised and a lumpsum tax should be added in order to retrieve these additional money holdings gradually (see the proof in the Appendix for all the details).

An example are the following taxes that spread the fiscal burden over all dates

$$
\tau_{t}(y)=\theta_{t}+\tilde{p}_{t}\left[\lim \sup q_{t}\left(y_{t-1}-y_{t}\right)-\lim y_{t}\right]^{+}
$$

We assume that $\tilde{p}$ and the lump-sum component $\theta$ are such that $\sum_{t=1}^{\infty} \theta_{t}<\infty, \tilde{p} \in \ell_{++}^{1}$, $\|\tilde{p}\|_{1}=1$. Moreover, we require $\lim q_{t} \tau_{t}(y)=0$. This is achieved if $\lim \tilde{p}_{t} q_{t}=0\left(\tilde{p}_{t}\right.$ tends to 
zero faster than $\left.p_{t}\right)$ and $\lim q_{t} \theta_{t}=0$. The presence of the lump-sum tax $\theta$ may be useful to withdraw additional initial holdings that allow for an implementation with non-negative money balances.

Observe that both $\lim y_{t}<\infty$ and $\left[\lim \sup q_{t}\left(y_{t-1}-y_{t}\right)-\lim y_{t}\right]^{+}<\infty$ for a plan $y$ that was already accommodating a bounded consumption plan $x(y)$ in the sequential budget set when taxes were not levied (as $y_{0}^{i}-\lim y_{t}=p\left(x(y)-\omega^{i}\right)<\infty$, since $\left.p_{t} q_{t}=1\right)$.

These non-lump-sum taxes are invariant to changes in $y$ at a finite set of dates and, therefore, Euler conditions (9) hold. However, it may be hard to accept that the tax authorities would have such a perfect foresight and we can suppose instead that the accumulated taxes up to each date, $\sum_{s \leq t} \tau_{s}(y)$ to depend just on $\left(y_{1}, \ldots, y_{t}\right)$.

Recursive tax schedules are of the form $\tau_{t}(y)=\theta_{t}+\tilde{\tau}(y)$ where $\theta$ is lump-sum (satisfying again $\lim q_{t} \theta_{t}=0$ ) and the non-lump-sum component is (again for $\tilde{p}$ such that $\lim \tilde{p}_{t} q_{t}=0$ ) such that

$$
\sum_{s \leq t} \tilde{\tau}_{s}(y) \equiv\left[\Phi_{t}-y_{t}-a_{t}\right]^{+} \sum_{s \leq t} \tilde{p}_{s}
$$

where $\Phi_{t}=\left[q_{t}\left(y_{t-1}-y_{t}\right)\right]^{+}$is what the agent dishoards at date $t$.

The sequence $a$ is chosen so that $\tilde{\tau}\left(y^{i}\right)=0$ for some reference allocation $\left(y^{i}\right)_{i}$. Let $a_{t} \equiv \max _{i}\left[\Phi_{t}-y_{t}^{i}\right]^{+}+1 / t$. Notice that the function $\tilde{\tau}_{t}$ depends only on money balances at finitely many dates but at $y^{i}$, it has null derivative with respect to these positions. A monetary equilibrium $\left(q,\left(x^{i}, y^{i}\right)_{i}\right)$ should fulfill the additional requirement that the equilibrium money balances allocation is the reference allocation. Then, Euler conditions (9) hold. Taxes defined by (13) allow for tax rebates: $\tilde{\tau}_{t}(y)$ can be negative if $\left|\tilde{\tau}_{t}(y)\right| \leq \sum_{s \leq t-1} \tilde{\tau}_{s}(y)$, but the latter is always non-negative ${ }^{22}$.

Schedule (13) tends to tax, at a non-early date $t$, an agent that dishoards at that date more than the whole cost of carrying on cash up to that date (which is the cost of carrying

\footnotetext{
${ }^{22}$ The non-convexity in (13) is overcome by mapping (non-linearly ) the budget set into the convex set of an auxiliary economy without taxes and constraints (i) or (ii) (see the Appendix).
} 
on cash on top of the initial holdings $\left(-\sum_{s \leq t} p_{s} q_{s}\left(y_{s-1}-y_{s}\right)\right)$ plus the cost of the initial holdings $\left(p_{1} q_{1} y_{0}^{i}\right)$, so the sum is $\left.p_{t} q_{t} y_{t}=y_{t}\right)$. Recursive taxes are in fact taxes on the use of savings rather than on savings per se and, therefore, have a flavor of a capital gains tax: the benefit from disposing of money balances is being taxed if it exceeds the cost of carrying on cash up to then.

Once recursive taxes have been imposed, we see that the plans $y$ accommodating budget feasible bounded $x(y)$ are those for which $\lim y_{t}$ and $\lim \left[x_{t}(y)-\omega_{t}^{i}\right]^{+}$exist.

If we add lump-sum taxes $\theta_{t} \geq \tilde{p}_{t} \lim _{t} a_{t}$ (and give each consumer an additional money holding equal to $\left.\sum_{t=1}^{\infty} \theta_{t}\right)$, we see that a plan $y$ inducing bounded $x(y)$ has a finite $\sum_{t=1}^{\infty} \tau_{t}(y)$ which is greater or equal to $\left[\lim \left[q_{t}\left(y_{t-1}-y_{t}\right)\right]^{+}-\lim y_{t}\right]^{+}$. It is immediate to see that (13) satisfies item (A) of Condition $\mathrm{C}^{23}$.

We may want to strengthen our assumptions on the tax schedule.

Condition C3: taxes are such that if all agents are impatient, an efficient monetary equilibrium must have money supply going to zero.

This assumption holds for taxes given by Equations (12) or (13), but would not hold if $A>0$ were added to the expressions inside square brackets in Equations (12) or (13) (although condition $\mathrm{C} 2$ would still hold). Under C3, any additional money holdings that might be needed to avoid short sales should be retrieved through appropriate lump-sum $\operatorname{taxes}^{24}$. We already knew that under C2 taxes are non-distortionary in terms of shortrun actions, in the sense that the Euler conditions that would hold without taxes (given by (9)) are still necessary for optimality when taxes are introduced. When C3 holds, on top of $\mathrm{C} 2$, taxes are non-distortionary in terms of long-run actions, in the sense that the

\footnotetext{
${ }^{23}$ We could have defined instead $\Phi_{t}=\sup { }_{s \leq t} q_{s}\left(y_{s-1}-y_{s}\right)$, for which $x(y)$ is bounded when lim $y_{t}$ exists (inducing a tax series greater or equal to $\left.\left[\sup _{t} q_{t}\left(y_{t-1}-y_{t}\right)-\lim y_{t}\right]^{+}\right)$. Item (A) is satisfied when $\operatorname{LIM}^{A D}$ is the Banach limit.

${ }^{24}$ Whereas by adding $A>0$ we could dispense with the lump sump taxes but would introduce a floor on money holdings.
} 
transversality conditions that would hold without taxes (given in Remark 2, for efficient allocations), together with its implications for the asymptotics of money supply (idem), must still hold when taxes are introduced.

Proposition 3. (Non-distortionary taxes) Let $\left(\left(x^{i}, y^{i}\right)_{i}, q\right)$ be a monetary equilibrium, for given initial holdings $\left(y_{0}^{i}\right)_{i}$ and taxes $\tau($.$) satisfying \boldsymbol{C 2}$. The transversality conditions, $\delta^{-} U^{i}\left(x^{i}, c\left(y^{i}(n)\right) \geq 0\right.$ and $\delta^{+} U^{i}\left(x^{i}, c\left(y^{i}(n)\right) \leq 0\right.$, are the same that would hold in the absence of taxes (and in the presence or not of constraints (i) or (ii)) if and only if taxes satisfy assumption $\mathbf{C 3}$. The result in Theorem 2 still holds under $\mathbf{C 3 .}$

Example 3 will show that in the absence of taxes satisfying C2 inefficient equilibria exist, where consumers' sequential problems have finite optimum since the allocations are implemented (using Proposition 2) with supergradients for which there are no asymptotic gains from dishoarding. The taxes proposed in C2 should not be seen as being required for the existence of any sequential equilibria. They are instead the efficient taxes on savings, in the sense that they implement efficient allocations.

\subsection{On money supply}

When agents are wary there may be multiple monetary implementations. Let us denote by $\mu^{i}+\nu^{i L}$ the pure charge of the supergradient of $U^{i}$ at $x^{i}$ which takes the highest value on the net trade of agent $i$. It is shown in the proof of Theorem 2 that, if $\mu^{i}$ is collinear with the countably additive component $p$ of the AD price $\pi$ and C3 holds, but (after rescaling utility so that $\left.\left\|\mu^{i}\right\|=\|p\|\right)$ we have $\nu^{A D}\left(x^{i}-\omega^{i}\right)<\nu^{i L}\left(x^{i}-\omega^{i}\right)$, then there is a monetary equilibrium with a positive limiting money supply. Let us explore this. To simplify, let us assume that preferences are differentiable along the canonical directions (and let us rescale the utility functions so that these canonical marginal derivatives $\mu_{t}^{i}$ are such that $\left.\left\|\mu^{i}\right\|=\|p\|\right)$. 




Figure 1: The marginal loss from undoing the savings $\left(b \cdot\left(x^{i}-\omega^{i}\right)\right)$ exceeds the AD price of the savings $\left(\pi \cdot\left(x^{i}-\omega^{i}\right)=0\right)$

Definition 3. We say that agent $\mathrm{i}$ is particularly wary at the $\mathrm{AD}$ allocation $\left(x^{i}\right)_{i}$ if agent's i marginal loss from reverting the savings policy (the left derivative of $U^{i}$ at $x^{i}$ along the direction $\left.x^{i}-\omega^{i}\right)$ is greater than the value that the $\mathrm{AD}$ support price assigns to this savings policy $\left(\pi\left(x^{i}-\omega^{i}\right)\right)$.

This non-differentiability is intrinsic to wary preferences, due to the diversity of generalized limits that can be taken for the pure charges of the supergradients. When $\delta^{-} U^{i}\left(x^{i}, x^{i}-\omega^{i}\right)>\pi\left(x^{i}-\omega^{i}\right)$, the consumer values the impact of the reversal of the savings policy differently from the way the AD supporting price does. Figure 1 illustrates what that gap would look like in a 2-date economy, although the non-differentiability is somehow ad-hoc in finite horizon but has a sound reason to occur for wary infinite lived agents. AD equilibria requires the support prices to be in the intersection of the superdifferentials of all agents. The sequential monetary implementation can be achieved by having each agent checking first order effects according to some supergradient, and there is no reason why agents should be coordinating to have collinear supergradients.

Theorem 3. (Equilibrium with non-vanishing money supply) Under the assumptions of Theorem 2, suppose that marginal utilities are well defined at each date and that 
some consumer $i$ is particularly wary at $x^{i}$, then, even for taxes satisfying also condition C3, there is a monetary implementation of the AD equilibrium for which the money supply does not go to zero.

Under impatience and strictly positive consumption, what has been known as the strong version of Friedman's rule still holds for taxes satisfying C2 and C3 (just like it did for lump-sum taxes $\left.{ }^{25}\right)$. On the other hand, Theorem 3 says that when agents are wary the money supply does not have to go to zero at an efficient monetary equilibrium, which contradicts Friedman's strong rule. Theorem 3 does not imply, however, that any monetary implementation, under its assumptions, must have non-vanishing money supply: given an equilibrium $(x, y, q)$, there is $r \in \ell_{+}^{1}$, with $\|r\|=1$ and $\lim r_{t} q_{t}=0$, such that if $\tilde{y}_{t}^{i}=$ $y_{t}^{i}-\left(\sum_{s \leq t} r_{s}\right) \lim y^{i}$, then $(x, \tilde{y}, q)$ is also an equilibrium, due to the automatic adjustment in the non-lump-sum taxes. The same consumption bundles are attained without carrying on cash to infinity but by putting aside from consumption the same amount, now in the form of taxes.

An interesting case where the assumptions of Theorem 3 holds is described next. The pure charges of all supergradients of $U^{i}$ at $x^{i}$ have the same norm if $\lim _{n} \delta^{-} U^{i}\left(x^{i}, 1^{n}\right)=$ $\lim _{n} \delta^{+} U^{i}\left(x^{i}, 1^{n}\right)$, where $1_{t}^{n}=0$ for $t<n$ and $1_{t}^{n}=1$ otherwise. In such case, the pure charge of the left derivative along the direction $\left(x^{i}-\omega^{i}\right)$ has the largest generalized limit of $\left(x^{i}-\omega^{i}\right)$, which is $\lim \sup \left(x^{i}-\omega^{i}\right)$. This will be illustrated as we resume Examples 1 and 2. Then, some consumer will be particularly wary at the AD allocation (and the assumptions of Theorem 3 hold $)$ if $\lim \sup \left(x^{i}-\omega^{i}\right) \neq L I M^{A D}\left(x^{i}-\omega^{i}\right)$ for some agent, which is the case when not all net trades are converging.

Corollary 1. Under the assumptions of Theorem 2, if for all agents $i$, the marginal derivatives at infinity $\lim _{n} \delta U^{i}\left(x^{i}, 1^{n}\right)$ and at each date $\delta U^{i}\left(x^{i}, e_{t}\right)$ exist, then there is a monetary implementation of the AD equilibrium for which the money supply does not tend to zero if

\footnotetext{
${ }^{25}$ In fact, lump-sum taxes do not affect the necessary conditions for individual optimality and the result follows as in Proposition 5 in Pascoa et al. (2010).
} 
and only if agents' net trades are not all converging.

This corollary is also proven in the Appendix. The necessity is actually quite general and was known from Remark 2; sufficiency depends on the assumptions that were made.

Let us compare our results with what had been established in the literature. What is designated as Friedman's strong rule is a variation upon a claim made by Friedman (1969), although his claim actually just required a zero nominal interest rate and that, for that purpose, money supply should contract at a rate equal to the equilibrium real interest rate. Bewley's work $(1980,1983)$ on impatient preferences not satisfying Inada had already shown that when consumption is always positive, a constant money supply is inefficient, whereas a money supply decreasing to zero at a constant rate can be made efficient when combined with lump-sum taxes. Levine (1986) gave interesting examples of efficient non-vanishing money supply for impatient agents with linear utilities, where corner solutions were crucial for building up large money balances. These were complemented by results in Levine (1989) on non-vanishing money supply under differentiable preferences not satisfying Inada. Wary agents have an incentive to keep large money balances for a long-run hedging effect, and, in this case, Inada's conditions will not prevent the implementation of efficient monetary equilibrium with constant money supply, as our theorems assert and the examples will illustrate.

\subsection{Monetary equilibrium for endogenous discounting}

\section{Resuming Example 1}

We consider two-agent economies where preferences are as in Example 1 and endowments suffer shocks that alternate in sign along time but are not of the same magnitude. When one consumer gets a positive shock, the other suffers the symmetric negative shock. Money can be used to hedge against these shocks. Consumers would like to hold money forever (or at least, along some subsequence) in order to find a consumption path in between 
the upper and the lower endowment subsequences.

The utility function is as in Equation (4) with $u^{i}()=.\sqrt{ }$. and $\beta=6$. Take, for both agents, $\zeta_{t}=(1 / 2)^{t-1} \sqrt{1+1 / t}$. Endowments are $\omega_{t}^{i}=16 \frac{t+1}{t}+G_{t}^{i}$, where $G_{t}^{1}$ is given by $G_{t}^{1}=13$ if $t$ is even and $G_{t}^{1}=-11$ if $t$ is odd, and $G_{t}^{2}=-G_{t}^{1}$. Recall that the indeterminacy in the generalized limit considered in the AD price leads to a real indeterminacy in AD equilibrium allocations. Take the equilibrium allocation that results from using a Banach limit $B$. Consider the allocation $x_{t}^{i}=16 \frac{t+1}{t}$ and supergradients of the form $\pi^{i} c=\sum_{t=1}^{\infty}\left(\frac{1}{2}\right)^{t+2} c_{t}+\frac{3}{4} B(c)$. We normalize prices so that the coefficient of the Banach limit is one: $\pi=\frac{4}{3} \pi^{i}$ (AD Lagrange multipliers are $3 / 4$ ). Denote by $p$ the summable component of $\pi$, the deflator $p_{t}=\frac{4}{3} 2^{-t-2}$. The pair $\left(\left(x^{i}\right)_{i}, \pi\right)$ is an $\mathrm{AD}$ equilibrium, as $\mathrm{AD}$ budget equations hold since $\pi\left(G^{1}\right)=0$ follows from $B\left(G^{1}\right)=1$ and $p\left(G^{1}\right)=-1$.

For $y_{0}^{i}=9$, make $q_{t}=\frac{3}{4} 2^{t+2}$, the inverse of the deflator $p_{t}$. Let $z_{t}$ be the funds put aside by a consumer at date $t$, which will be decomposed as a sum of his money balances and the cumulated taxes on his money balances: $z_{t}=y_{t}+\sum_{s \leq t} \tau_{s}^{i}(y)$.

As the infimum $\underline{x}^{i}$ of consumption is never attained, the marginal utility at infinity $\lim _{n} \delta U^{i}\left(x^{i}, 1^{n}\right)$ exists (see Appendix E) and, therefore, the assumptions of Corollary 1 are satisfied. The implementation is achieved (as explained in detail in Appendix A) with $\left(z^{i}\right)_{i}$ if we (I) make $\lim _{t} z_{t}^{i}=\lim \sup \left(x^{i}-\omega^{i}\right)$, that is, the limiting cost of carrying on cash equals the marginal gain of hedging at infinity, given by the highest possible value that any pure charge of a supporting price can take on the net trade and (II) require all other plans $\hat{z}$ to satisfy $\lim _{t} \hat{z}_{t}^{i} \geq \lim \sup \left(x\left(\hat{z}^{i}\right)-\omega^{i}\right)$ (a limiting cost of funds not below the marginal gain at infinity).

Taxes are designed so that Condition (II) holds. A money holdings plan y pays accumulated taxes $\sum_{t=1}^{\infty} \tau_{t}^{i}(y)=\lim \sup \left(x\left(\hat{y}-\omega^{i}\right)-\lim _{t} y_{y}\right.$, which ensures (II).

The AD budget equation holds if $z_{0}^{i}=\lim _{t} z_{t}^{i}-B\left(x^{i}-\omega^{i}\right)$. Then, (I) implies that $z_{0}^{i}=\lim \sup \left(x^{i}-\omega^{i}\right)-B\left(x^{i}-\omega^{i}\right)$, that is, $\lim \sup \left(-G^{1}\right)-B\left(-G^{1}\right)=z_{0}^{1}$ and $\lim \sup \left(G^{1}\right)-$ $B\left(G^{1}\right)=z_{0}^{2}$, where $B\left(G^{1}\right)=1$. Since $\lim \sup \left(-G^{1}\right)=11$ and $\lim \sup \left(G^{1}\right)=13$ we must 
have $z_{0}^{1}=z_{0}^{2}=12$. So $z_{t}^{i}=12+\sum_{s=1}^{t} p_{s} G_{s}^{i}$ and short-sales are never done in equilibrium ${ }^{26}$. Then, $\lim z_{t}^{1}=11$ whereas $\lim z_{t}^{2}=13$.

Now, take $\theta=0$ and $y^{i}=z^{i}$ so that equilibrium cash balances are not taxed and money supply remains constant, which illustrates Theorem 1.

Actually, as $\lim z_{t}^{1}$ is different from $\lim z_{t}^{2}$ we could not make $\sum_{t=1}^{\infty} \theta_{t}=\lim z_{t}^{i}$ for all $i$, so that money supply would tend to zero. Impersonal taxes are incompatible with a limiting zero money supply, except in the symmetric case where $\lim \sup \left(x^{i}-\omega^{i}\right)$ is the same for all agents, as implied by Theorem 2 .

This example can be modified so that aggregate resources are not decreasing but, for any $t$, there exists some subsequent date where the aggregate endowment is lower than in $t$. Suppose that at even dates endowments follow increasing sequences and that at odd dates endowments are oscillating around a decreasing trend.

As a second remark, notice that the discount factors are a product of exponential and hyperbolic discounting. Preferences fail to be time-consistent, not as a consequence of $\beta^{i}$ being positive, but as a result of the somehow hyperbolic discounting that was assumed for convenience reasons. The example could be redone with longer computations (along the lines of Example 1 in (Araujo et al. (2011))) under exponential discounting and consumption plans that differ from the trend endowment.

\section{Resuming Example 2}

Consider two agents with utility functions of the form in Example 2 with $u^{i}(y)=\sqrt{y}$, $n^{i}=2, \eta_{t}^{i}=\left(\frac{1}{2}\right)^{t-1} \sqrt{1+1 / t}$ and

$$
\beta^{i}=3\left(8 \sum_{k=0}^{\infty} \frac{1}{2^{4^{k}}}-8 \sum_{k=0}^{\infty} \frac{1}{2^{4^{k}+2^{k}}}-12 \sum_{k=0}^{\infty} \frac{1}{4^{4^{k}}}+12 \sum_{k=0}^{\infty} \frac{1}{2^{2 \cdot 4^{k}+2^{k}}}\right) .
$$

The consumptions are defined as $\omega^{i}:=16 \frac{1+t}{t}+G_{t}^{i}$ where $G_{t}^{1}=6$ if $2^{2 k+1} \leq t \leq 2^{2(k+1)}-$

\footnotetext{
${ }^{26}$ In this example we did not need to increase initial money holdings by some amount $A$ to avoid shortsales.
} 
1 for $k=0,1, \ldots$ and $G_{t}^{1}=-4$ if $2^{2 k} \leq t \leq 2^{2 k+1}-1$ for $k=0,1, \ldots$, and $G_{t}^{2}=-G_{t}^{1}$ for all $t \in \mathbb{N}$. Take the equilibrium allocation $x_{t}^{i}=16 \frac{1+t}{t}$ that results from using in the AD price the pure charge, LIM, that in the net trade of the agent 1, takes the lowest value, $\liminf _{t}\left(x_{t}^{1}-\omega_{t}^{1}\right)$. The AD price is given by $\pi(c)=\sum_{t=0}^{\infty}\left(\frac{1}{2}\right)^{t+2}+\frac{\beta}{8} \operatorname{LIM}(\phi(c))$ where $\phi$ : $\ell_{+}^{\infty} \rightarrow \ell_{+}^{\infty}$ such that $\phi(c)_{t}=\frac{1}{t} \sum_{k=1}^{t} c_{k}$. Since $\phi$ is Frechet differentiable, using the result of the chain rule of the Clark subdiferential, we have that all pure charges in the subdifferential of the agent have the same norm, and also that the left derivative in the direction of the net trade coincides with the lim sup of the net trade, since the value of $\phi$ in the net trade of the first agent is $\phi(4,-6,-6,4,4,4,4,-6,-6,-6,-6,-6,-6,-6,-6,4,4,4,4,4,4, \ldots)=$ $(4,-1,-8 / 3,-1,0,2 / 3,8 / 7,1 / 4,-4 / 9,-1,-11 / 6,-28 / 13,-17 / 7,-8 / 3, \ldots)$. This implies that we can implement with $z^{1}=10$ and $z_{0}^{2}=0$ (by the same argument as in Example 1) and taxes defined as in Example 1.

\subsection{On inefficient equilibria when there are no taxes}

\section{Example 3}

Consider n economy economy with two agents $i=1,2$ whose preferences are given by (4), where $u^{i}(x):=\log (x), \delta_{t}=1 / 2^{t}$ and $\beta$ will be specified below.

$$
\omega_{t}^{i}= \begin{cases}8+2^{-t} & \text { if } t \text { and } i \text { are even or odd simultaneously, } \\ 2 & \text { if } t \text { is even and } i \text { is odd, or conversely. }\end{cases}
$$

There is an $\mathrm{AD}$ equilibrium $(\pi, x)$ where $x_{t}^{i}=5+2^{-t-1}$ for each $i$, and $\pi(c):=$ $\sum_{t=1}^{\infty} \frac{1}{2^{t}} \frac{c_{t}}{5+2^{-t-1}}+\nu(c)$, for a pure charge $\nu$ defined by $\nu(\cdot)=\frac{\beta}{5} \operatorname{LIM}(\cdot)$ where LIM is a generalized limit such that $\operatorname{LIM}\left(x^{1}-\omega^{1}\right)=\lim \sup \left(x^{1}-\omega^{1}\right)$, provided that $\beta$ is given as follows

$$
\beta:=\frac{5}{3}\left(\sum_{k \geq 1} \frac{2}{4^{k}}\left(5+4^{-k}\right)^{-1}\left(3+4^{-k}\right)-\sum_{k \geq 1} \frac{1}{4^{k}}\left(5+\frac{4^{-k}}{2}\right)^{-1}\left(3+\frac{4^{-k}}{2}\right)\right)>0
$$


Now, let us construct an inefficient equilibrium $\left(\left(\tilde{x}^{i}, \tilde{x}^{i}\right)_{i}, q\right.$ without deflation, $q_{1}=\cdots=$ $q_{t}=q$, where agent 1 holds no money in all even dates $\left(\tilde{z}_{2 k}^{1}=0\right.$ for $\left.k \in \mathbb{N}\right)$ and agent 2 holds no money in all odd dates $\left(\tilde{z}_{2 k-1}^{1}=0\right.$ for $\left.k \in \mathbb{N}\right), \tilde{z}_{0}^{1}=0$ and $\tilde{z}_{0}^{2}=6 \beta$. Let $\left(\mu_{t}^{i}\right) \in \partial U^{i}\left(\tilde{x}^{i}\right)$ and satisfying the FOC of each agent at $\tilde{x}^{i}$. That is, for each $t$ and $i$ even or odd simultaneously,

$$
\delta_{t} u^{i^{\prime}}\left(\tilde{x}_{t}^{i}\right)=\mu_{t}^{i}=\mu_{t+1}^{i}=\delta_{t+1} u^{\prime}\left(\tilde{x}_{t+1}^{i}\right)+\gamma_{t+1} \beta u_{i}{ }^{\prime}\left(\underline{\tilde{x}}^{i}\right)=\left(\delta_{t+1}+\gamma_{t+1} \beta\right) u^{i^{\prime}}\left(\underline{\tilde{x}}^{i}\right)
$$

Therefore, $\left(\tilde{x}_{t}^{i}\right)_{i, t},\left(\gamma_{t}^{i}\right)_{i, t}$ and $q$ are such that satisfy:

- $\sum_{t=1}^{\infty} \gamma_{t}^{i}=1$ where $\gamma_{t}^{i} \geq 0$ with $\gamma_{t}^{i}=0$ for $t$ and $i$ both odd or both even;

- $\inf _{t} \tilde{x}_{t}^{1} \equiv \underline{\tilde{x}}^{1}=\tilde{x}_{2 k}^{1}$ for all $k \in \mathbb{N}$ and for agent 1 , and $\inf _{t} \tilde{x}_{t}^{2} \equiv \underline{\tilde{x}}^{2}=\tilde{x}_{2 k-1}^{2}$ for all $k \in \mathbb{N}$ and for agent 2 , by choosing $q<1 /(2 \beta)$;

- $\tilde{x}_{t}^{i}=\omega_{t}^{i}-6 \beta q$ and $\tilde{x}_{t+1}^{i}=\omega_{t+1}^{i}+6 \beta q$ for each $t$ and $i$ even or odd simultaneously, then, by Equation (15), $\delta_{t} u^{i^{\prime}}\left(\omega_{t}^{i}-6 \beta q\right)=\left(\delta_{t+1}+\gamma_{t+1}\right) u^{i^{\prime}}\left(\omega_{t+1}^{i}+6 \beta q\right)$ for each $t$ and $i$ even or odd simultaneously.

The plan $\left(\tilde{x}^{i}, \tilde{z}^{i}\right)$ is optimal for agent $i$. To see this, notice that this infimum attained infinitely many times (in all $t$ for which $\tilde{z}_{t}^{i}=0$ ) and the plan satisfies the sufficient conditions mentioned in Lemma 2, for a supergradient whose pure charge is zero. In fact, the transversality condition (10) is satisfied: $\lim _{t} \mu_{t}^{i} q_{t} \tilde{z}_{t}^{i}=q \lim _{t} \mu_{t}^{i} \tilde{z}_{t}^{i}=0$, as there is no deflation and $\tilde{z}^{i}$ takes a positive value, $6 \beta$, only at dates where the respective subsequence of $\mu^{i}$ is falling to zero. On the other hand, the condition (11) becomes $\lim _{t} \mu_{t}^{i} q_{t} z_{t} \geq 0$, which is trivially satisfied for any $z \geq 0$.

However, it can be noticed that the equilibrium under sequential budget constraints just constructed is inefficient since the marginal rates of substitution are not equal for the two agents in all pairs of dates (more precisely, no supergradient of one agent is collinear with a supergradient of the other agent). If we impose the taxes mentioned in Subsection 4.1, it is possible to implement the $\mathrm{AD}$ equilibrium mentioned above which is clearly Pareto efficient. 
If such taxes, of the recursive form (13), were levied upon the inefficient equilibrium plans, agent 1 would pay a tax $x_{t}^{1}-\omega_{t}^{1}-y_{t}=6 \beta q$ on even dates (dates when the agent dishoarded more than the cost of carrying on cash up to that date) and zero taxes on odd dates. These taxes would displace the inefficient plans and guide the consumers toward efficient savings plans. However, if we just add lump-sum taxes, the outcome will not be Pareto efficient.

\section{Concluding Remarks}

The paper is related to the work in Araujo et al. (2011) on sequential implementation of $\mathrm{AD}$ allocations using long-lived assets paying dividends, but differs from it in three crucial aspects. First, efficient allocations are now being sequentially implemented by introducing taxes that discourage inefficient savings, rather than by imposing portfolio constraints. We believe this approach is quite novel.

Second, the two papers differ in what the bubble is. Before, for portfolio constraints involving the $\mathrm{AD}$ pure charge, the bubble was just equal to the value that the $\mathrm{AD}$ price pure charge takes at the dividends sequence (see, Theorem 2 in Araujo et al. (2011)), but in the case of money such value would be zero. Moreover, it was also shown (see Proposition 6, in Araujo et al. (2011)), that for more general portfolio constraints (using possibly other generalized limits when evaluating asymptotic net trades), the bubble would still be determined in the same way (and, therefore, equal to zero in the case of fiat money) whenever agents' net trades were all converging. However, we show that, in the implementation with taxes proposed in the current paper, for non-converging AD net trades, the initial money holdings scaled up by the bubble coincide with the difference between the highest value that the pure charge of a supergradient can take at the net trade and the corresponding value for the AD price pure charge. The non-differentiability of preferences, inherent to wariness, plays now a crucial role ${ }^{27}$.

\footnotetext{
${ }^{27}$ see the proof of Lemma 4 in the Appendix.
} 
Third, we have now general results for wary preferences and endogenous discounting, being no longer focused on the specific form driven by the $\epsilon$-contamination capacity.

Having chosen to focus on monetary implementations, we came up with the interesting observation that money supply does not have to go to zero. Wary agents can use persistent positive money balances to hedge against endowments shocks at far away dates. An optimal positive limit in the money supply is not a consequence of imposing money floors or peculiar portfolio constraints. We just assume the usual no-short-sales constraint on money together with a fiscal policy that taxes inefficient savings plans and corrects what would be an insatiable demand for precautionary liquidity in a deflationary context (an instance of a problem already noticed by Friedman and Bewley).

Finally, it should be pointed out that time consistency is compatible with wariness. As an example, when the series of discounted utilities describes a time-consistent behavior (say, under exponential discounting), then adding a term dealing with the infimum of the utilities makes the consumer wary and could introduce an inconsistency but it does not in equilibrium as long as the infimum is not attained in finite time, which is precisely the case we are interested in, so that $\mathrm{AD}$ prices exhibit pure charges.

\section{APPENDIX}

\section{A Proof of Theorem 1}

Proof. $\left(x_{t}\right)_{t \in \mathbb{N}} \ggg 0$ implies that the marginal utility in $x_{t}$ of the function $u$ is uniformly bounded from above and below, that is, $0<m \leq u^{\prime}\left(x_{t}\right) \leq M<\infty$ implying that the 
condition of uniform convergence can be written as $\lim _{t} \sup _{\delta \in C}\left\{\sum_{s \geq t} \delta_{s}\right\}=0$. Therefore,

$$
\begin{aligned}
0 & \geq \lim _{n \rightarrow \infty} \lim _{h \rightarrow 0^{-}} \frac{-1}{h}\left(U\left(x+h \mathbb{1}_{E_{n}}\right)-U(x)\right) \\
& =\lim _{n \rightarrow \infty} \lim _{h \rightarrow 0^{-}} \frac{-1}{h}\left(\inf _{m \in C}\left\{\sum_{t=1}^{n-1} \delta_{t} u\left(x_{t}\right)+\sum_{t=n}^{\infty} \delta_{t} u\left(x_{t}+h\right)\right\}-\inf _{m \in C}\left\{\sum_{t=1}^{\infty} \delta_{t} u\left(x_{t}\right)\right\}\right) \\
& \geq \lim _{n \rightarrow \infty} \lim _{h \rightarrow 0^{-}} \frac{-1}{h}\left(\inf _{m \in C}\left\{\sum_{t=n}^{\infty} \delta_{t}\left(u\left(x_{t}+h\right)-u\left(x_{t}\right)\right)\right\}\right) \\
& \geq \lim _{n \rightarrow \infty} \lim _{h \rightarrow 0^{-}} \frac{-1}{h}\left(\inf _{m \in C}\left\{\sum_{t=n}^{\infty} \delta_{t}(M h+o(h))\right\}=-M \lim _{n \rightarrow \infty} \sup _{m \in C}\left\{\sum_{t=n}^{\infty} \delta_{t}\right\}=0\right.
\end{aligned}
$$

which concludes proves that the left derivative is 0 , for $h \rightarrow 0^{+}$is analogous.

\section{B Proof of Theorem 2}

We construct an auxiliary economy where inter-temporal transfers of wealth are done by trading a no-dividends asset in constant positive net supply, not subject to taxes but subject to portfolio constraints. We denote positions in this asset by $z$ (these will be related to money balances by $z_{t}=y_{t}+\sum_{s \leq t} \tau_{s}^{i}(y)$, which implies that $\left.z_{t-1}^{i}-z_{t}^{i}=y_{t-1}^{i}-y_{t}^{i}-\tau_{t}(y)\right)$. In the auxiliary economy, budget constraints are given by (8).

Consider the supergradient whose pure charge $\nu^{i L}$ takes the highest value on the direction of the net trade ${ }^{28}$. That is, $\nu^{i L}$ is such that $\delta^{-} U^{i}\left(x^{i} ; x^{i}-\omega^{i}\right)=\left(\mu^{i}+\nu^{i L}\right)\left(x^{i}-\omega^{i}\right)$. If $\mu^{i}$ is collinear with the countably additive part $p$ of the AD price $\pi$, then we use the supergradient $\mu^{i}+\nu^{i L}$ in the procedure proposed by Proposition 2. Otherwise, we can always use the supergradient collinear with $\pi \equiv p+\nu^{A D}$. This suggests the following portfolio constraint:

$$
\lim \mu_{t}^{i} q_{t} z_{t} \geq \nu^{i L}\left(x(z)-\omega^{i}\right)
$$

Let $B^{A}\left(q, y_{0}^{i}, \omega^{i}\right)$ be the set of plans $(x, z)$ satisfying (8) and (16).

\footnotetext{
${ }^{28}$ This allows us to illustrate the multiplicity of equilibria and prepare for the proof of Theorem 3 .
} 
Definition 4. A vector $\left(q,\left(x^{i}, z^{i}\right)_{i \in \mathcal{I}}\right) \in \mathbb{R}_{+}^{\infty} \times\left(\ell_{+}^{\infty} \times \mathbb{R}_{+}^{\infty}\right)^{I}$ is an equilibrium for the auxiliary economy with initial holdings $\left(z_{0}^{1}, \ldots, z_{0}^{I}\right)$ if $\left(x^{i}, z^{i}\right) \in \operatorname{argmax}\left\{U^{i}(x):(x, z) \in\right.$ $\left.B^{A}\left(q, y_{0}^{i}, \omega^{i}\right)\right\} ; \sum_{i=1}^{I} x^{i}=\sum_{i=1}^{I} \omega^{i}$ and $\sum_{i=1}^{I} z_{t}^{i}=\sum_{i=1}^{I} z_{0}^{i} \forall t \in \mathbb{N}$.

Lemma 4. If $\left(\left(x^{i}\right)_{i}, \pi\right)$ be an $A D$ equilibrium such that $x^{i} \ggg 0$, there exist $z_{0}^{i}$ that implement $\left(x^{i}\right)_{i}$ as an equilibrium for the auxiliary economy, possibly with short-sales.

Proof. Notice that the $\mathrm{AD}$ budget equation holds as an equality for a plan $z^{i}$ when $\lim _{t} p_{t} q_{t} z_{t}^{i}-\nu^{A D}\left(x\left(z^{i}\right)-\omega^{i}\right)=z_{0}^{i} p_{1} q_{1}$. We choose $z_{0}^{i}$ so that $\frac{1}{\rho^{i}} \nu^{i L}\left(x\left(z^{i}\right)-\omega^{i}\right)-\nu^{A D}\left(x\left(z^{i}\right)-\right.$ $\left.\omega^{i}\right)=z_{0}^{i} p_{1} q_{1}$, where $\rho^{i}$ is the AD Lagrange multiplier of agent $i$. We can actually take $p_{t} q_{t}=1$. By Proposition 2, the portfolios $z^{i}$ that satisfy (16) given $z_{0}^{i}$ and $x^{i}$, will implememt the $\mathrm{AD}$ equilibrium allocation $\left(x^{i}\right)_{i}$ for $\left(\omega^{i}\right)_{i}$.

Let us map back into the original sequential economy. Suppose sequential implementation without taxes was achieved with short sales under the constraint (16), with $p_{t} q_{t}=1$. If $z$ takes negative values at some dates, we can find money holdings $Z_{0}^{i}=z_{0}^{i}+\tilde{A}$ such that the equilibrium positions $z_{t}^{i}$ can be replaced by non-negative money balances. We have the freedom of either shifting up the portfolio plans by $\tilde{A}$ or introducing lump-sum taxes $\theta$ that retrieve the additional initial holdings gradually (lightly at the finitely many dates where $z_{t}^{i}$ was negative), or a combination of both ${ }^{29}$.

Let us proceed by introducing taxes that replace the portfolio constraints.

Lemma 5. The non-negative plans $Z^{i}$ given by $Z_{t}^{i}=z_{t}^{i}+\tilde{A}-\sum_{s \leq t} \theta_{s}$ for $t \geq 1$, will implement the same efficient allocation if portfolio constraints are replaced by personal taxes $\tau^{i}$ satisfying, for any portfolio plan $Z, \sum_{t=1}^{\infty} \tau_{t}^{i}(Z)=\sum_{t=1}^{\infty} \theta_{t}+\left[\nu^{i}\left(q_{t}\left(Z_{t-1}-Z_{t}\right)\right)-\right.$ $\lim Z+A]^{+}$, where $\theta_{t}=\tilde{p}_{t}(\tilde{A}-A)$.

\footnotetext{
${ }^{29}$ Notice that if we choose to shift $z^{i}$ up by $\tilde{A}$, then $Z^{i}$ does not satisfy the second transversality condition of Remark 2 (the direction $Z^{i}(n)$ is not left admissible for the constraint $\lim _{t} \mu_{t} q_{t} z_{t} \geq \nu(x(z)-\omega)+\tilde{A}$, which should replace (11), together with $\lim _{t} \mu_{t} q_{t} z_{t}^{i} \geq \nu\left(x^{i}-\omega\right)+\tilde{A}$ replacing $\left.(10)\right)$
} 
Proof. In fact, for $x_{t}^{i}(Z) \equiv q_{t}\left(Z_{t-1}-Z_{t}-\tau_{t}^{i}(Z)\right)$ we have $\sum_{t=1}^{\infty} \tau_{t}^{i}(Z) \geq \nu^{i}\left(x^{i}(Z)-\omega^{i}\right)-$ $\lim Z_{t}+\tilde{A}$. Let $z_{t}=Z_{t}-\tilde{A}+\sum_{s \leq t} \tau_{s}^{i}(Z)$, then $\lim _{t} z_{t} \geq \nu^{i}\left(x(z)-\omega^{i}\right)$. That is, the definition of taxes ensures that any plan $Z$ has an image $z$ satisfying constraint (11). As we already knew that (10) holds, it follows that $Z^{i}$ is optimal, for the initial holding $Z_{0}^{i}=z_{0}^{i}+\tilde{A}$, and taxes are levied in equilibrium only if we choose to have lump-sum taxes removing (all or part of) $\tilde{A}$.

Now, in order to define impersonal taxes we will increase taxes and also the initial holdings of money. Let $\gamma^{i}:=\lim \sup \left(x^{i}-\omega^{i}\right)-\nu^{i}\left(x^{i}-\omega^{i}\right) \geq 0$. We make $y_{0}^{i}=Z_{0}^{i}+\gamma^{i}$. Denoting by $q\left(y_{-}-y\right)$ the sequence with general term $q_{t}\left(y_{t-1}-y_{t}\right)$, for any portfolio plan $y$ we define the following tax

$$
\gamma_{t}^{i}(y)= \begin{cases}\left(\lim \sup q\left(y_{-}-y\right)-\nu^{i}\left(q\left(y_{-}-y\right)\right)\right) \tilde{p}_{t} & \text { if } \lim y \leq \nu^{i}\left(q\left(y_{-}-y\right)\right)+A \\ \left.\left[\lim \sup q\left(y_{-}-y\right)\right)-\lim y+A\right]^{+} \tilde{p}_{t} & \text { otherwise }\end{cases}
$$

Then, the impersonal taxes satisfy

$$
\left.\sum_{t=1}^{\infty} \tau_{t}(y)=\sum_{t=1}^{\infty}\left(\tau_{t}^{i}(y)+\gamma_{t}^{i}(y)\right)=\sum_{t=1}^{\infty} \theta_{t}+\left[\lim \sup q\left(y_{-}-y\right)\right)-\lim y+A\right]^{+} .
$$

Lemma 6. Given the equilibrium plans $\left(Z^{i}\right)_{i}$ for the economy with just personal taxes $\tau^{i}$, the plans $y_{t}^{i}=Z_{t}^{i}+\gamma^{i}-\sum_{s \leq t} \tilde{p}_{s}\left(\lim \sup \left(x^{i}-\omega^{i}\right)-\nu^{i}\left(x^{i}-\omega^{i}\right)\right)$ constitute an equilibrium for the economy with impersonal taxes.

Proof. Let us see first that the proposed plans $y^{i}$ are in the budget set with impersonal taxes. For the proposed plans, $\lim y^{i}=\lim Z^{i}=\nu^{i}\left(q\left(y_{-}-y\right)\right)+\tilde{A}-\sum_{t=1}^{\infty} \theta_{t}$ and therefore $\tau^{i}\left(y^{i}\right)=\sum_{t=1}^{\infty} \theta_{t}$. Moreover, as $\lim _{t} q_{t} \tau_{t}(y)=0$ we have $\lim \sup q\left(y_{-}^{i}-y^{i}\right)=\lim \sup \left(x^{i}-\omega^{i}\right)$ and $\nu^{i} q\left(y_{-}^{i}-y^{i}\right)=\nu^{i}\left(x^{i}-\omega^{i}\right)$. So, $\gamma_{t}^{i}\left(y^{i}\right)=\tilde{p}\left(\lim \sup \left(x^{i}-\omega^{i}\right)-\nu^{i}\left(x^{i}-\omega^{i}\right)\right)$. Then, $y_{t-1}^{i}-y_{t}^{i}-\tau_{t}\left(y^{i}\right)=Z_{t-1}^{i}-Z_{t}^{i}-\theta_{t}$, which implies that $y^{i}$ still accommodates $x^{i}$ in the sequential budget equations.

Now, $\left(Z^{i}\right)_{i}=\left(z^{i}+A-\sum_{s \leq t} \theta_{s}\right)_{i}$ is also an equilibrium for the auxiliary economy, with constraints (11). To show that $y^{i}$ is optimal for the economy with impersonal taxes, it 
suffices to show that any plan $y$ for consumer $i$ in the economy with impersonal taxes induces a plan $z$ for $i$ in the auxiliary economy with constraints (11). We look for $z$ such that $y_{t-1}-y_{t}-\tau_{t}(y)=z_{t-1}-z_{t}$, then we have

$$
z_{t}=y_{t}-\left(\gamma^{i}-\sum_{s \leq t} \gamma_{s}^{i}(y)\right)+\sum_{s \leq t} \tau_{s}^{i}(y)
$$

Then, $\lim z_{t}=\lim y_{t}+\sum_{t=1}^{\infty} \tau_{t}^{i}(y) \geq \nu^{i}\left(q\left(y_{-}-y\right)\right)+\tilde{A}\left(\right.$ since $\left.\sum_{t=1}^{\infty} \theta_{t}+A=\tilde{A}\right)$. Now, $\nu^{i}\left(q\left(y_{-}-y\right)\right)=\nu^{i}\left(q\left(z_{-}-z\right)\right)$ since $\lim q_{t} \tau_{t}(y)=0$. Hence, constraint (16) holds.

This concludes the Proof of Theorem 2.

The proof of Proposition 3 follows from the proof of Theorem 2 since, under C2, taxes satisfy $\mathrm{C} 3$ if and only if $A=0$, which is also the necessary and sufficient condition for $\lim y_{t}^{i}$ to be equal to the limit of the equilibrium portfolio $z^{i}$ of the auxiliary economy (for which the assumptions in Remark 2 hold).

\section{Proof of Theorem 3}

Notice that for taxes to satisfy condition C3 we make $A=0$ and we get $\lim y^{i}=\nu^{i}\left(x^{i}-\right.$ $\left.\omega^{i}\right)$. If there is at least one agent $j$ such that $\nu^{j}\left(x^{j}-\omega^{j}\right)$ for some supergradient $\mu^{j}+$ $\nu^{j}$, with $\mu^{j}$ collinear with $p$ and $\nu^{j}\left(x^{j}-\omega^{j}\right) / \rho^{j}>\nu^{A D}\left(x^{j}-\omega^{j}\right)$, then the monetary equilibrium generated (according to Proposition 2) by using this supergradient for $j$ and supergradients collinear with $p+\nu^{A D}$ for all other agents, will have money supply converging to $\sum_{i \neq j} \nu^{A D}\left(x^{i}-\omega^{i}\right)+\nu^{j}\left(x^{j}-\omega^{j}\right) / \rho^{j}>0$.

Finally, for each $i$ let $Y_{t}^{i}=y_{t}^{i}-\sum_{s \leq t} r_{s} \lim y^{i}$, where $r \in \ell_{+}^{1},\|r\|_{1}=1$ and $r_{t} q_{t} \rightarrow 0$. Now, $\left[\nu^{i} q\left(Y_{-}^{i}-Y^{i}\right)-\lim Y^{i}+A\right]^{+}=\left[\nu^{i} q\left(y_{-}^{i}-y^{i}\right)+\lim q_{t} r_{t} \lim y^{i}+A\right]^{+}=\nu^{i} q\left(y_{-}^{i}-y^{i}\right)+A$ and therefore $\sum_{t=1}^{\infty} \tau_{t}\left(Y^{i}\right)=\sum_{t=1}^{\infty} \tau_{t}\left(y^{i}\right)+\lim y^{i}$. Actually, for taxes given by (12) it is immediate to see that $\tau_{t}\left(Y^{i}\right)=\tau_{t}\left(y^{i}\right)+\tilde{p}_{t} \lim y^{i}$. For other tax schedules (say given by (13) or its variants (a) and (b) considered in a footnote) we pick $r_{t}=\frac{\tau_{t}\left(Y^{i}\right)-\tau_{t}\left(y^{i}\right)}{\lim y^{i}}$. 
Then, $q_{t}\left(Y_{t-1}^{i}-Y_{t}^{i}-\tau_{t}\left(Y^{i}\right)\right)=q_{t}\left(y_{t-1}^{i}-y_{t}^{i}+r_{t} \lim y^{i}-r_{t} \lim y^{i}-\tau_{t}\left(y^{i}\right)\right)=q_{t}\left(y_{t-1-y_{t}^{i}}^{i}\right.$ $\left.\tau_{t}\left(y^{i}\right)\right)$ and, therefore, $Y^{i}$ is optimal for agent $i$.

\section{Proof of Corollary 1}

We just need to rule out that $\operatorname{LIM}^{A D}\left(x^{i}-\omega^{i}\right)=\lim \sup \left(x^{i}-\omega^{i}\right)$, for any $i$. Adding across agents, $0=\sum_{i} \lim \sup \left(x^{i}-\omega^{i}\right)$. Say it is agent 1 whose net trade $x^{1}-\omega^{1}$ does not converge. Now, $\lim \sup \left(x^{1}-\omega^{1}\right)=-\sum_{i \neq 1} \lim \sup \left(x^{i}-\omega^{i}\right)=\sum_{i \neq 1} \lim \inf \left(\omega^{i}-x^{i}\right) \leq \lim \inf \left(x^{1}-\omega^{1}\right)$, a contradiction.

\section{E On Example 1 and Directional Derivatives of the Utility}

We show here that for a utility function $\mathrm{U}$ of the form given by (4), if $z^{*}$ is an optimal portfolio plan in $B^{A}\left(q, y_{0}^{i}, \omega^{i}\right)$ (defined in Subsection 8.2.1) such that, at $x^{*}:=x\left(z^{*}\right) \ggg 0$, we have inf $x^{*}$ not attained and $\lim _{s} x_{s}^{*}=\inf _{s} x_{s}^{*}$, then

$$
\delta^{-} U\left(x^{*}\right)\left(x^{*} ; x^{*}-\omega^{i}\right)=\mu\left(x^{*}-\omega^{i}\right)+\alpha \lim \sup \left(x^{*}-\omega^{i}\right)
$$

for $\alpha>0$ equal to the norm of the pure charge component of a supergradient of $U$ at $x^{*}$, where $\mu$ is given by $\mu_{t}=\zeta_{t} u^{\prime}\left(x_{t}^{*}\right)$.

We will estimate $\lim _{r \rightarrow 0} \frac{1}{r}\left[U \circ x\left(z^{*}+r z^{*}\right)-U \circ x\left(z^{*}\right)\right]$. Consider the direction $\Delta \in$ $\ell^{\infty}$ given by $\Delta_{t}=q_{t} z_{t-1}^{*}-q_{t} z_{t}^{*}$. Notice that $\lim _{r \rightarrow 0} \frac{1}{r} \sum_{t \geq 1} \zeta_{t}\left[u\left(x_{t}^{*}+r \Delta_{t}\right)-u\left(x_{t}^{*}\right)\right]=$ $\sum_{t \geq 1} \zeta_{t} \lim _{r \rightarrow 0} \frac{1}{r}\left[u\left(x_{t}^{*}+r \Delta_{t}\right)-u\left(x_{t}^{*}\right)\right]=\sum_{t \geq 1} \zeta_{t} u^{\prime}\left(x_{t}^{*}\right) \Delta_{t}$. So, what we still need to do is to estimate $\lim _{r \uparrow 0} \frac{1}{r} \beta\left[\inf _{t} u\left(x_{t}^{*}+r \Delta_{t}\right)-\inf _{s} u\left(x_{s}^{*}\right)\right]$, which is $\delta^{-} \inf _{t} u\left(x^{*}, \Delta\right)$, the left-derivative of the function $\inf _{t} u($.$) along the direction \Delta$ evaluated at $x^{*}$.

Observe that there exists $\chi>0$ such that $\forall r \in(-\chi, 0)$ the following holds: $(1+r) z^{*}>0$ is a non-negative plan, $x\left(z^{*}+r z^{*}\right)$ satisfies (16) and $x\left(z^{*}+r z^{*}\right)=x^{*}+r\left(x^{*}-\omega\right) \ggg 0$.

ClaIm: $\quad \lim _{r \uparrow 0} \frac{1}{r}\left[\inf _{t} u\left(x_{t}^{*}+r \Delta_{t}\right)-\inf _{t} u\left(x_{t}^{*}\right)\right]=u^{\prime}\left(\underline{x}^{*}\right) \lim \sup _{t} \Delta_{t}$ 
Proof. Let us suppose that $x_{t}^{*}$ converges to $\underline{x}^{*}=\inf x^{*}$, then $\lim _{r \uparrow 0} \frac{1}{r}\left[\inf _{t} u\left(x_{t}^{*}+r \Delta_{t}\right)-\right.$ $\left.u\left(\underline{x}^{*}\right)\right]$ since $\inf ():. \ell^{\infty} \rightarrow \mathbb{R}$ is a concave function.

Fixed $r \in(-\chi, 0)$ and given $\epsilon>0$, it is valid for all $\tau$ large enough that $(1 / r)\left[\inf _{t} u\left(x_{t}^{*}+\right.\right.$ $\left.\left.r \Delta_{t}\right)-u\left(\underline{x}^{*}\right)\right]+\epsilon=(-1 / r)\left[u\left(\underline{x}^{*}\right)-\epsilon r-\inf _{t} u\left(x_{t}^{*}+r \Delta_{t}\right)\right] \geq(-1 / r)\left[u\left(x_{\tau}^{*}\right)-u\left(x_{\tau}^{*}+r \Delta_{\tau}\right)\right] \geq$ $u^{\prime}\left(x_{\tau}^{*}\right) \Delta_{\tau}$. Making $\tau \rightarrow \infty$ we get $(1 / r)\left[\inf _{t} u\left(x_{t}^{*}+r \Delta_{t}\right)-u\left(\underline{x}^{*}\right)\right]+\epsilon \geq \lim \sup _{t} u^{\prime}\left(x_{t}^{*}\right) \Delta_{t}=$ $u^{\prime}\left(\underline{x}^{*}\right) \lim \sup _{t} \Delta_{t}$, for an arbitrary $\epsilon>0$.

To prove the reverse inequality, notice that, under the hypothesis, $\delta U\left(x^{*} ; \mathbb{u}(n)\right)=$ $\sum_{t>n} \zeta^{t} u^{\prime}\left(x_{t}^{*}\right)+\beta u^{\prime}\left(\underline{x}^{*}\right)$ and, therefore, any supergradient has a pure charge component with norm $\beta u^{\prime}\left(\underline{x}^{*}\right)$ by Lemma 1. Hence, for any supergradient $T$ of $U$ at $x^{*}$ we have $T(\Delta)=$ $\sum_{t \geq 1} \zeta_{t} u^{\prime}\left(x_{t}^{*}\right) \Delta_{t}+\beta u^{\prime}\left(\underline{x}^{*}\right) \operatorname{LIM}(\Delta)$, for some generalized limit LIM. So, $\delta^{-} \inf _{t} u\left(x^{*}, \Delta\right) \leq$ $u^{\prime}\left(\underline{x}^{*}\right) \lim \sup _{t} \Delta_{t}$.

Now, if there is a subsequence $S$ such that $\Delta_{t} \geq 0, \inf _{S} x_{t}^{i}=\underline{x}^{*}$ and $\lim \sup _{S} \Delta_{t}=$ $\limsup \Delta_{t}$, the left derivative on the direction $\left\{\Delta_{t}\right\}_{t}$ is $u^{\prime}\left(\underline{x}^{*}\right) \lim \sup _{t} \Delta_{t}$, which concludes the proof.

\section{Acknowledgements}

We gratefully acknowledge the financial support from CAPES, CNPq and FAPERJ in Brazil and from FCT/FEDER (project EGE-ECO/114496/2009) and NOVA FORUM in Portugal. We are grateful to comments by seminar audiences at UCLA, the University of Chicago Money Workshop, the Harvard-MIT Theory seminar, 2nd BWGT at USP and Sorbonne Workshop in Ec. Theory 2014.

\section{References}

A. Araujo, Lack of Pareto Optimal Allocations in Economics with Infinitely Many Commodities: the Need for Impatience, Econometrica 53 (1985) 455-461.

A. Araujo, R. Novinski and M. Pascoa, General Equilibrium, Wariness and Efficient Bubbles, Journal of Economic Theory 53 (2011) 785-811. 
D. Backus, B. Routledge and S.Zin, Exotic Preferences for Macroeconomists, NBER working paper 10597, 2004.

T. Bewley, Existence of Equilibrium in Economies with Infinitely Many Commodities, Journal of Economic Theory 4 (1972) 514-540.

T. Bewley, The Optimum Quantity of Money. in Models of Monetary Economics, ed. by J. Kareken and N.Wallace, Federal Reserve Bank, Minneapolis, 1980.

T. Bewley, A Difficulty with the Optimum Quantity of Money, Econometrica 51 (1983), 1485-1504.

D. Brown and L. Lewis, Myopic Economic Agents, Econometrica 49 (1981) 359-368.

J. Dow and S. Werlang, Uncertainty Aversion, Risk Aversion and the Optimal Choice of Portfolio, Econometrica 60 (1992), 197-204.

N. Dunford and J. Schwartz, Linear Operators, Part I, Interscience, New York, 1958.

M. Friedman, Essays in positive economics, University of Chicago Press, 1953.

M. Friedman, The Optimum Quantity of Money, in The Optimum Quantity of Money and Other Essays, Aldine, Chicago, 1969.

P. Y. Geoffard, Discounting and Optimizing: Capital Accumulation Problems as Variational Minmax Problems, Journal of Economic Theory 69 (1996), 53-70.

I. GilboA and D. Schmeidler, Maxmin Expected Utility with a Non-Unique Prior, Journal of Mathematical Economics 110 (1989) 605-39.

L. HAnsen, Exotic Preferences for Macroeconomists: Comment, NBER Macroeconomics Annual 19 (2004) 391-405.

L. Hansen and T. Sargent, Robust Control and Model Uncertainty, The American Economic Review 91 (2001) 60-66.

L. Hansen and T. Sargent, Robustness, Princeton University Press, New Jersey, 2008.

U. Khan and M. Stinchсомb, Planning for the Long Run: Programming with Patient, Pareto Responsive Preferences, Working paper, 2016.

T. Kehoe, D. Levine and M. Woodford, The Optimum Quantity of Money Revisited, in P. Dasguptha, D. Gale, O. Hart and E. Maskin (eds.), The Economic Analysis of Markets and Games: Essays in Honor of Frank Hahn, MIT Press, 501-526, 1992.

T. Koopmans, Stationary Ordinary Utility and Impatience, Econometrica 28 (1960) 287-309.

D. Levine, Borrowing Constraints and Expansionary Policy, UCLA unpublished, 1986.

D. Levine, Asset Trading Mechanisms and Expansionary Policy, Federal Reserve Bank, Minneapolis, working paper 385, 1988.

D. Levine, Efficiency and the Value of Money, Review of Economic Studies 56(1989) 77-88. 
M. MARINACCI, An axiomatic approach to complete patience and time invariance. Journal of Economic Theory, 83 (1998) 105-144.

A. Mas-Colell and W. Zame, Equilibrium theory in infinite dimensional spaces, Handbook of mathematical economics 4, 1835-1898, 2010.

M. Pascoa, M. Petrassi and J. Torres-Martinez, Fiat Money and the Value of Binding Portfolio Constraints, Economic Theory 46 (2010) 189-209.

L. RAut, Myopic topologies on general commodity spaces, Journal of Economic Theory 39 (2) (1986) 358-367.

P. Samuelson, An Exact Consumption-Loan Model of Interest with or without the Social Contrivance of Money, The Journal of Political Economy 66 (1958) 467-82.

D. Schmeidler, Subjective Probability and Expected Utility without Additivity, Econometrica 57 (1989) 571-587.

H. Uzawa, Time Preference, the Consumption Function and Optimal Asset Holdings, in J.N.Wolfe. eds. Value, Capital and Growth: Papers in Honor of Sir John Hicks. Chicago IL: Aldine Publishing Company, 1968.

C. von Weizsacker, Existence of Optimal Programs of Accumulation for an Infinite Time Horizon, Review of Economic Studies 32 (1965) 85-104.

M. Woodford, The Optimum Quantity of Money, in B. Friedman and F. Hahn (eds.), Handbook of Monetary Economics, vol. II, ch. 20, North Holland, Amsterdam, 1990. 